Article

\title{
Facile Access to Fe(III)-Complexing Cyclic Hydroxamic Acids in a Three-Component Format
}

\author{
Evgeny Chupakhin ${ }^{1,2}$, Olga Bakulina ${ }^{1}$, Dmitry Dar'in ${ }^{1} \mathbb{C}$ and Mikhail Krasavin ${ }^{1,2, *}$ \\ 1 Institute of Chemistry, Saint Petersburg State University, 199034 Saint Petersburg, Russia; \\ chupakhinevgen@gmail.com (E.C.); o.bakulina@spbu.ru (O.B.); d.dariin@spbu.ru (D.D.) \\ 2 Institute of Living Systems, Immanuel Kant Baltic Federal University, 236016 Kaliningrad, Russia \\ * Correspondence: m.krasavin@spbu.ru; Tel.: +7-931-3617872; Fax: +7-812-428-6939
}

Academic Editors: Richard A. Bunce, Philippe Belmont, Wim Dehaen and Eugene Babaev Received: 12 February 2019; Accepted: 25 February 2019; Published: 28 February 2019

\begin{abstract}
Cyclic hydroxamic acids can be viewed as effective binders of soluble iron and can therefore be useful moieties for employing in compounds to treat iron overload disease. Alternatively, they are analogs of bacterial siderophores (iron-scavenging metabolites) and can find utility in designing antibiotic constructs for targeted delivery. An earlier described three-component variant of the Castagnoli-Cushman reaction of homophthalic acid (via in situ cyclodehydration to the respective anhydride) was extended to involve hydroxylamine in lieu of the amine component of the reaction. Using hydroxylamine acetate and O-benzylhydroxylamine was key to the success of this transformation due to greater solubility of the reagents in refluxing toluene (compared to hydrochloride salt). The developed protocol was found suitable for multigram-scale syntheses of $\mathrm{N}$-hydroxy- and $\mathrm{N}$-(benzyloxy)tetrahydroisoquinolonic acids. The cyclic hydroxamic acids synthesized in the newly developed format have been tested and shown to be efficient ligands for $\mathrm{Fe}^{3+}$, which makes them suitable candidates for the above-mentioned applications.
\end{abstract}

Keywords: iron complexation; cyclic hydroxamic acids; Castagnoli-Cushman reaction; homophthalic acid; hydroxylamine acetate; azeotropic distillation; cyclodehydration

\section{Introduction}

The ability of cyclic hydroxamic acids ( $N$-hydroxylactams) to chelate metal ions in general—and $\mathrm{Fe}^{3+}$ in particular-defines their utility in drug design [1]. One principal avenue in this regard is based on the recognition of the similarity of synthetic cyclic hydroxamic acids to bacterial siderophores-special metabolites excreted by bacteria to scavenge and deliver iron for the microorganism's nutritional needs [2]. Conjugating such moieties to antibiotics helps shuttle those inside bacteria and thus overcome resistance of the latter to the antibacterial agent [3]. Besides said application in resistance-free antibiotic design, selective, non-toxic iron chelators are needed as treatments for hereditary iron overload disease [4]. Unfortunately, streamlined and multicomponent methods to prepare $\mathrm{N}$-hydroxylactams are lacking. Among the existing synthetic methods for hydroxamic acid, intramolecular nucleophilic cyclization onto $O$-protected acyclic hydroxamic acids [5], nitroso moiety insertion in cyclic ketones [6], and ring-closing methathesis of bis-olefinic hydroxamic acids [7] can be mentioned.

Imines $\mathbf{1}$ are known to condense with $\alpha-\mathrm{C}-\mathrm{H}$ anhydrides of dicarboxylic acids 2 to give polysubstituted lactams 3 [8]. In the recent literature, this powerful reaction has been regarded as a name reaction - the Castagnoli-Cushman reaction (CCR) [9] - to acknowledge its origination from the research efforts of Neil Castagnoli and Mark Cushman over 40 years ago [10,11]. Homophthalic anhydride (HPA, 4) is one of the most frequently employed anhydrides in this reaction, which normally gives rise to trans-configured tetrahydroisoquinolonic (THIQ) acids 5 (Figure 1) [12,13]. These have 
a documented utility in medicinal chemistry (in therapeutic areas such as cancer [14], malaria [15], and diabetes [16]).

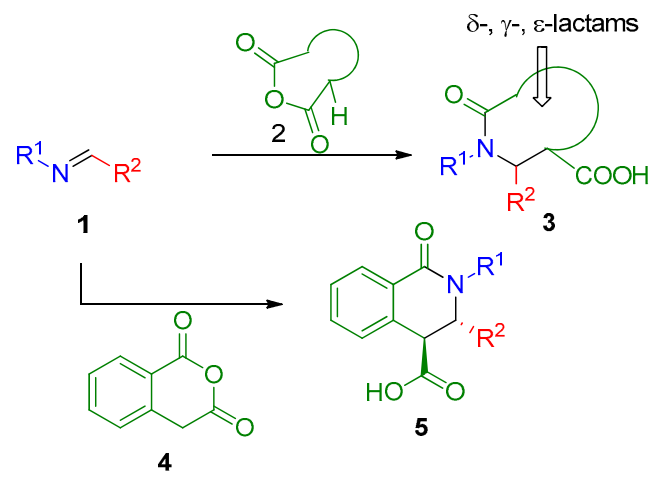

Figure 1. The Castagnoli-Cushman reaction (CCR).

Recently, we successfully replaced the imine component in the CCR of 4 with oximes 6 and obtained, after $24 \mathrm{~h}$ reflux in toluene, good to excellent yields of the respective $N$-hydroxy THIQ acids 7 [17] which are representative of cyclic hydroxamic acids. The forcing conditions required in order to obtain 7 were in sharp contrast with ambient temperature normally sufficient for the preparation of 5 . This was justified [17] by the need to re-generate HPA (4) from the initial, rapidly formed $O$-acylation product $7^{\prime}$ (Scheme 1).

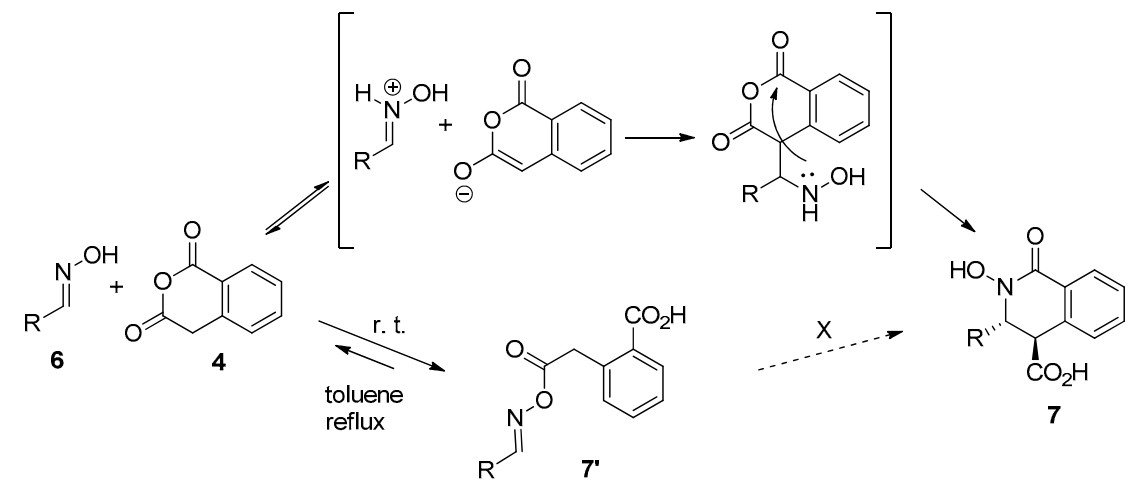

Scheme 1. Reaction of oximes 6 with HPA (4) and its mechanistic interpretation [15].

More recently, we employed refluxing toluene for in situ dehydration of homophthalic acid which allowed, for the first time, preparing THIQ acids 5 in a true multicomponent format (i.e., by mixing an amine, an aldehyde and homophthalic acids) without a need for preliminary imine synthesis or the use of hydrolytically prone HPA (Scheme 2) [18].

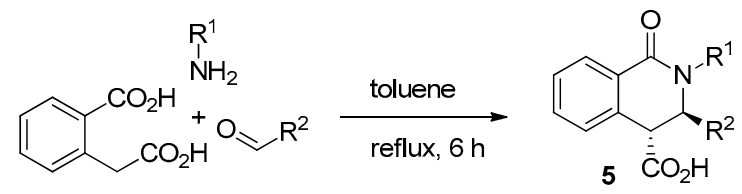

Scheme 2. Synthesis of THIQ acids 5 in a three-component format from homophthalic acid [18].

These findings encouraged us to investigate the preparation of $N$-hydroxy THIQ acids 7 from homophthalic acid and oximes and possible applicability in this case of the same three-component format as presented in Scheme 2. Herein, we present the results of these findings and present characterization of compounds 7 with respect to their iron-binding properties, which validates them as potential candidates for iron overload disease treatment or the design of siderophore-based constructs for antibiotic delivery. 


\section{Results and Discussion}

The initial reaction of oxime 6 a with homophthalic acid in toluene proved rather encouraging and yielded, after $16 \mathrm{~h}$ reflux and cooling to room temperature, 72\% of the desired product 7a [17] (Scheme 3). The reaction proceeded via formation of HPA (4), which was supported by the results of a separate experiment, where homophthalic acid was refluxed in toluene with azeotropic removal of water to form corresponding anhydride in quantitative yield. This experiment can be also regarded as a new method for the preparation of homophthalic anhydride.

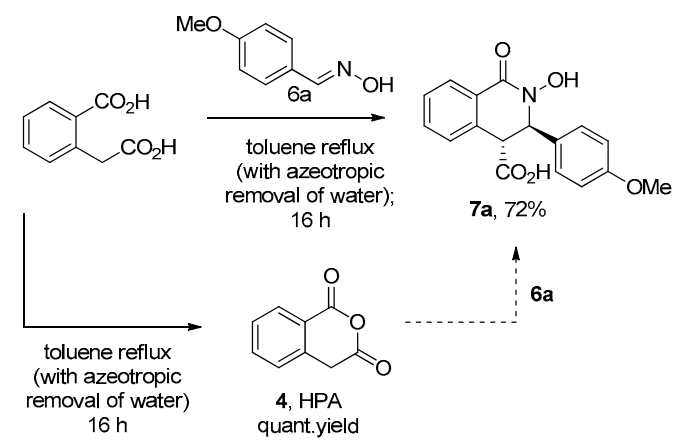

Scheme 3. Two-component preparation of $7 \mathbf{a}$ from homophthalic acid.

An attempt to perform the same reaction by mixing homophthalic acid with $p$-anisaldehyde and hydroxylamine hydrochloride in presence of pyridine (1 equiv.) did not lead to the formation of 7a. Instead, the only product discernible by ${ }^{1} \mathrm{H}-\mathrm{NMR}$ (Nuclear Magnetic Resonance) analysis of the reaction mixture was tetracyclic adduct formed, presumably, via the earlier described [19] Perkin-Michael domino transformation (Scheme 4).

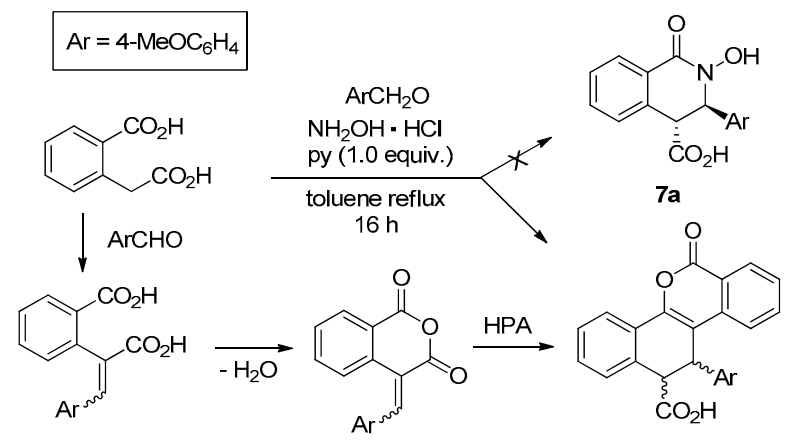

Scheme 4. Attempted three-component preparation of 7a from hydroxylamine hydrochloride (see ref. [19] for more mechanistic insight).

The observed complication is likely the result of the inefficient formation of the intermediate oxime 6 in the non-polar reaction medium (due to low solubility in it of hydroxylamine hydrochloride) and the ready participation of free $p$-anisaldehyde in the route leading to tetracyclic adduct. To circumvent this obstacle, we attempted to prepare hydroxylamine acetate and use it in the same transformation. We anticipated that in addition to improved solubility of the acetate salt in toluene, this transformation would not require the use of a base as acetic acid would be conveniently removed from the reaction medium by azeotropic distillation with the solvent. Interestingly, hydroxylamine acetate has been seldom [20] employed for the preparation of oximes despite the obvious advantage of not having to use any base (as is the case with the usual hydroxylamine hydrochloride). The hydrochloride salt of hydroxylamine was converted to its acetate by the action of sodium acetate and reacted with $p$-anisaldehyde and homophthalic acid in refluxing toluene over $24 \mathrm{~h}$. To our delight, on cooling to r. t., a thick precipitate formed which was isolated by filtration to provide $65 \%$ yield of compound 7a. The same reaction was attempted at reflux in benzene and xylenes. While the former conditions 
led to $<15 \%$ conversion over $24 \mathrm{~h}$, the latter gave an appreciable amount of $N$-deoxygenation product. Considering this and the results of the reaction monitoring at different time points, $24 \mathrm{~h}$ reflux in toluene was considered to be optimal for the preparation of compound 7a. Thus, these conditions were extended to the preparation of a new series of $N$-hydroxy THIQ acids $7 \mathbf{a}-\mathbf{m}$ (Scheme 5).

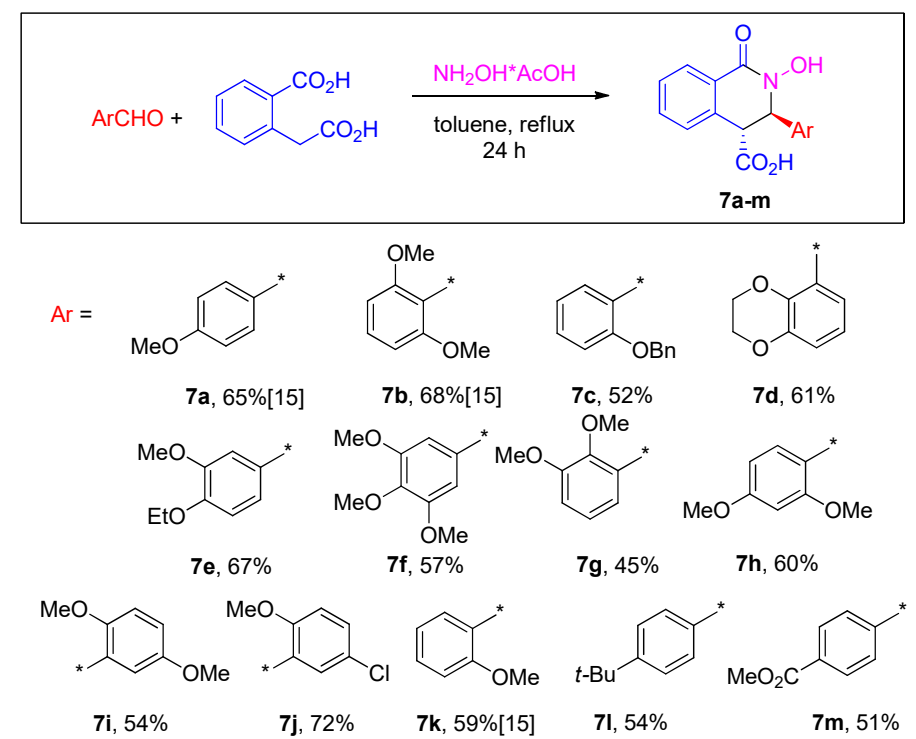

Scheme 5. Three-component synthesis of $N$-Hydroxy THIQ acids $7 \mathbf{a}-\mathbf{m}$.

An interesting result was obtained in an attempt to involve $o$-salicylaldehyde in the same reaction. When all the aldehyde starting material was consumed (according to HPLC (High Performance Liquid Chromatography) analysis of the reaction mixture), only a trace amount of anticipated compound was detected. The major product identified and isolated from the reaction mixture was coumarin 8. The same reaction run without hydroxylamine acetate gave identical yield of 8 (67\%). It likely that the reaction (described earlier but not interpreted from the mechanistic viewpoint [21]) involves acylation of the phenolic hydroxy group followed by intramolecular Knoevenagel reaction (Scheme 6). Involvement of $\mathrm{O}$-acetyl salicylaldehyde in the same reaction led to de-acetylation and the formation of 8 in $50 \%$ yield.

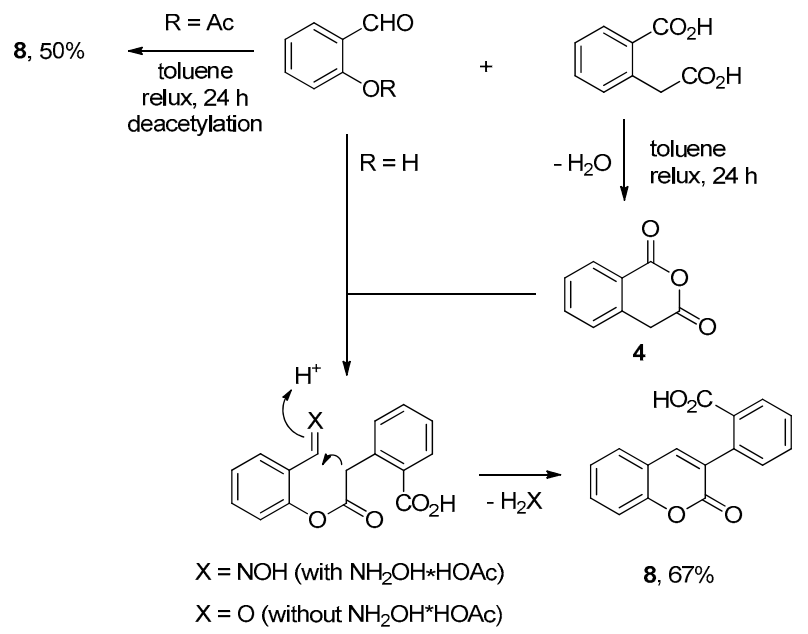

Scheme 6. Involvement of unprotected $o$-salicaldehyde in the reaction with homophthalic acid.

The reaction generally worked very well with electron-rich aromatic aldehydes and gave good yields of respective $N$-hydroxy THIQ acids 7a-k (Scheme 5). Substrates without alkoxy groups (such as tert-butyl-, methoxycarbonyl-, fluoro-, bromo-, and nitro-benzaldehydes,) surprisingly, gave no desired 
product under these conditions. Increasing the concentration of reactants from $0.1 \mathrm{M}$ to $1 \mathrm{M}$ and the reaction time from 24 to $48 \mathrm{~h}$, allowed us to slightly increase the reaction scope by involving 4-(tert-butyl)benzaldehyde and 4-(methoxycarbonyl)benzaldehyde in the developed approach and to prepare corresponding $N$-hydroxy THIQ acids 71 and $7 \mathbf{m}$ in good yields (54 and 51\% respectively). However, still the reaction was not applicable to more electron-poor substrates.

The ${ }^{1} \mathrm{H}$ - and ${ }^{13} \mathrm{C}-\mathrm{NMR}$ analysis of corresponding reaction mixtures showed that in case of electron-poor aldehydes the major reaction products are nitriles (formed by dehydration of oximes). To prevent this side reaction, we replaced hydroxylamine acetate with O-benzylhydroxylamine (Scheme 6). To our delight this allowed to involve previously unreactive electron-poor aldehydes (even 4-fluoro- and 4-nitro-benzaldehydes). Following this new modified protocol (also performed at $1 \mathrm{M}$ concentration of reactants) seven novel $\mathbf{N}$-benzyloxy THIQ acid derivatives $\mathbf{9 a}-\mathbf{g}$ have been prepared in high and good yields (Scheme 7). For all prepared compounds $\mathbf{7 a - m}$ and $\mathbf{9 a}-\mathbf{g}$ trans-configuration was concluded from ${ }^{3} \mathrm{~J}_{\mathrm{HH}}$ coupling constant values $(\sim 1-2 \mathrm{~Hz})$ between protons at positions $\mathrm{C} 3$ and $\mathrm{C} 4$, which is consistent with our previous data [17].

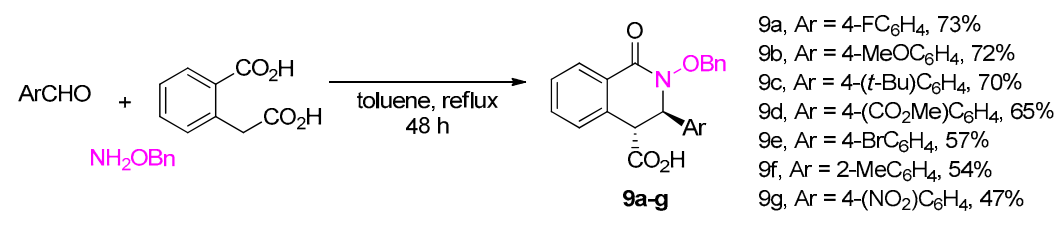

Scheme 7. Three-component synthesis of $N$-Benzyloxy THIQ acids 9.

Additionally, two types of post-modifications have been investigated for the prepared compounds 7 and 9: decarboxylation (Scheme 8) and debenzylation (Scheme 9). Attempts to perform decarboxylation of compound 7a under previously reported conditions [22] lead to formation of complex mixture of products resulting from side reactions of oxidation and dehydration (supported by HRMS (High Resolution Mass Spectrometry) and ${ }^{1} \mathrm{H}-\mathrm{NMR}$ data) (Scheme 8). At the same time, decarboxylation can be smoothly conducted for $O$-benzylated compound $\mathbf{9 b}$ providing compound 10 in $77 \%$ yield, thus demonstrating another advantage of $O$-benzyloxime-based protocol for preparation of N-hydroxy THIQ acids derivatives.

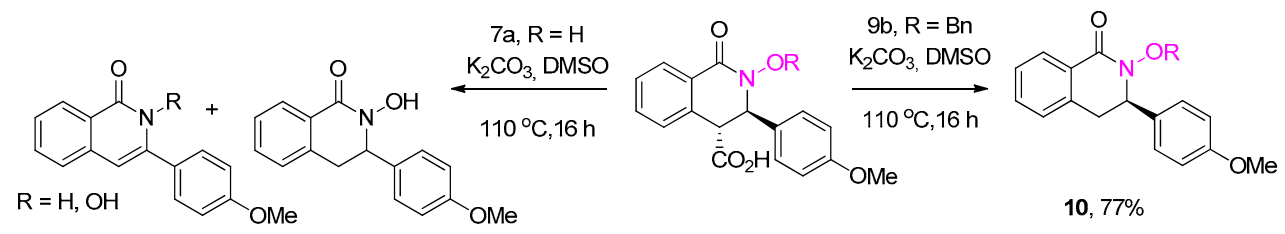

Scheme 8. Decarboxylation of compounds $9 a$ and $9 b$.

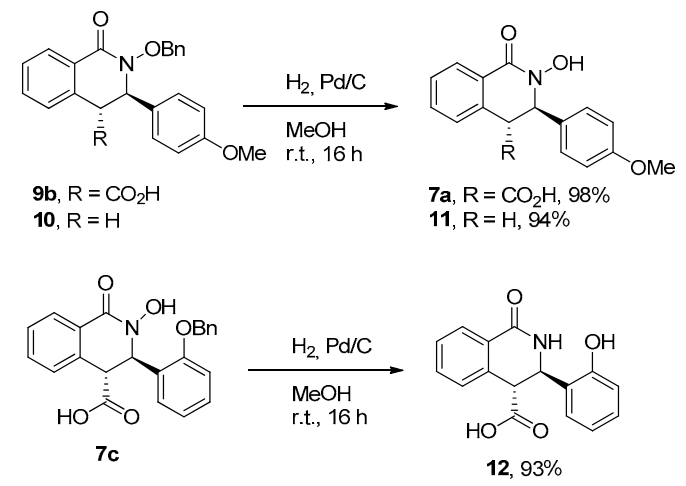

Scheme 9. Debenzylation of compounds $9 b, 10$, and $7 c$.

We also have investigated the possibility of removal of benzyl protective group from prepared compounds (Scheme 9). N-Benzyloxy THIQ acids $\mathbf{9 b}$ and $\mathbf{1 0}$ were successfully converted to 
corresponding $\mathrm{OH}$-hydroxamic acids 7a and $\mathbf{1 1}$ via hydrogenolysis under standard conditions with excellent yields. Surprisingly, attempted $O$-debenzylation of compound $7 \mathrm{c}$ only gave the product of $\mathrm{N}$-dehydroxylation 12 (Scheme 9).

Compounds 7 synthesized using the developed procedure are direct analogs of bacterial siderophores [23] and, therefore, can be potentially regarded as new agents for iron overload disease therapy and as precursors for the design of "Trojan horse" antibiotics [24] against drug resistant bacteria. Therefore five selected compounds- $\mathbf{7} \mathbf{d}, \mathbf{f}-\mathbf{h}, \mathbf{j}$ which represent different substitution patterns in 3-aryl moiety (4 types of di-substituted and one tri-substituted), were tested for iron(III) binding properties using mole-ratio method to prove this assumption (Figure 2a,b). Upon addition of ferric nitrate to solution of ligand 7 in aqueous methanol a color change from colorless to purple was observed. This corresponds to formation of $\mathrm{Fe}(\mathrm{III})-7$ complex, which is characterized by a new absorption band with maximum around $500 \mathrm{~nm}$ (Figure 2b). All ligands 7 in contrast to their complexes with iron do not absorb in the visible region. Representative example of UV-Vis spectrum of free ligand 7 and its changes upon addition of $\mathrm{Fe}^{3+}$ ions is shown on Figure 2a for compound $\mathbf{7 d}$. These spectra for other tested compounds 7 are reported in ESI (Electronic Supporting Information, Figures S1-S3). In all cases two isosbestic points were observed around 470 and $600 \mathrm{~nm}$ at $\mathrm{C}_{\mathrm{Fe}}=0.5-5 \times 10^{-4} \mathrm{M}$.

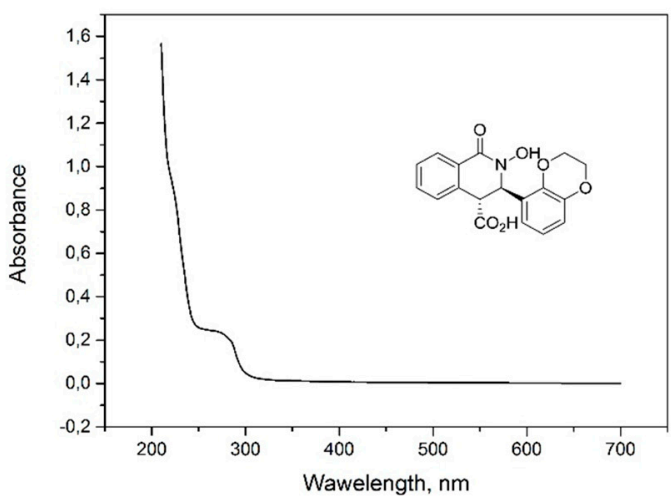

(a)

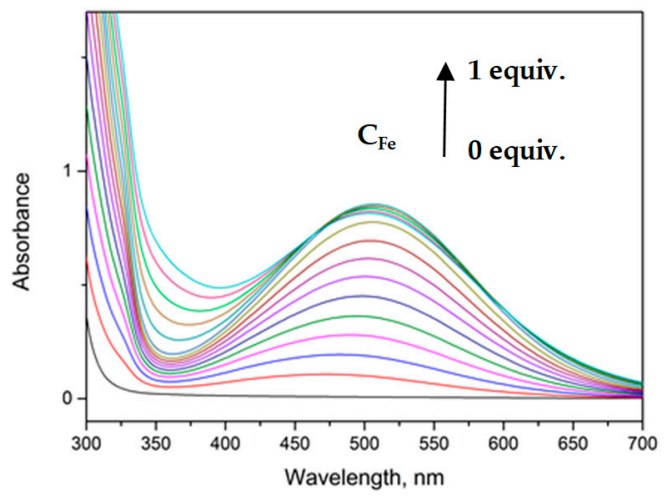

(b)

Figure 2. (a) UV-Vis absorbance spectrum of compound $7 \mathbf{d}$ in methanol $\left(\mathrm{C}=5 \times 10^{-5} \mathrm{M}\right)$; (b) UV-Vis absorbance spectrum of compound $7 \mathbf{d}\left(\mathrm{C}_{\mathrm{L}}=5 \times 10^{-4} \mathrm{M}\right.$, aq. $\left.\mathrm{MeOH} 85 \%\right)$ in the presence of increasing concentration of $\mathrm{Fe}^{3+}\left(\mathrm{C}_{\mathrm{Fe}}=0.25-5 \times 10^{-4} \mathrm{M}\right)$.

Applying the mole-ratio method allowed us to determine not only the stoichiometry of observed complexes but also the associated binding constants $[17,25]$. Absorbance at characteristic wavelength was plotted as function of $\left[\mathrm{Fe}^{3+}\right] /[$ ligand] ratio to give the curves shown in Figure 3a. The binding process involves two equilibria-the first one corresponding to 1:1 complex, and the second one to 1:2 complex. Stepwise formation constants $K_{1}$ and $K_{2}$ were estimated in the range of $10^{5}-10^{7} \mathrm{M}^{-1}$ and $10^{4}-10^{5} \mathrm{M}^{-1}$, respectively. These values were calculated using computer nonlinear curve-fitting of the absorbance values taken from experimental mole-ratio plots on Figure 3 a to previously derived Equations (1)-(3) describing formation of $\mathrm{ML}_{2}$ complex (See Material and Methods Section for details). The example of such fitting (compound $7 \mathbf{d}$ ) is presented on Figure 3b. The respective plots with curve-fitting for other tested compounds $\mathbf{7 f}-\mathbf{h}, \mathbf{j}$ are presented in ESI (Figures S4-S8) The results on spectrophotometric investigation of iron(III) binding properties for compounds 7 are summarized in Table 1. The obtained $\mathrm{K} f$ values $\left(\sim 10^{10}-10^{11} \mathrm{M}^{-1}\right)$ were found to be in accordance with our previous results [17] for similar compounds. No significant dependence of $K_{f}$ values on substitution pattern was found. 


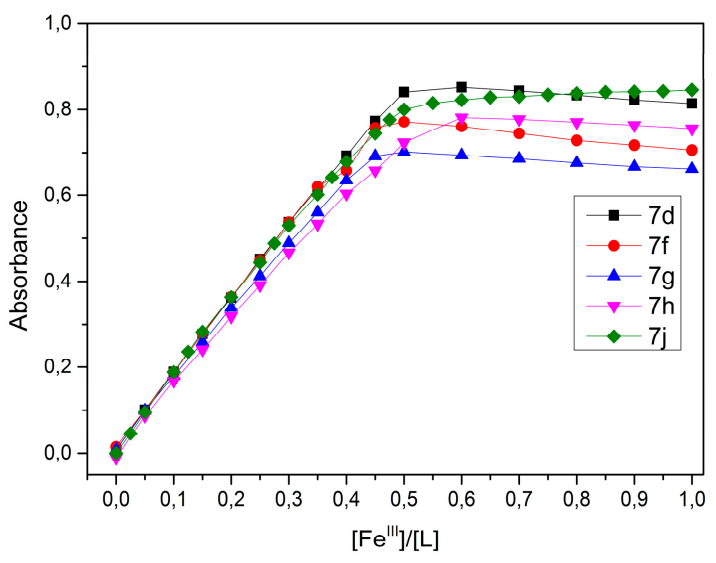

(a)

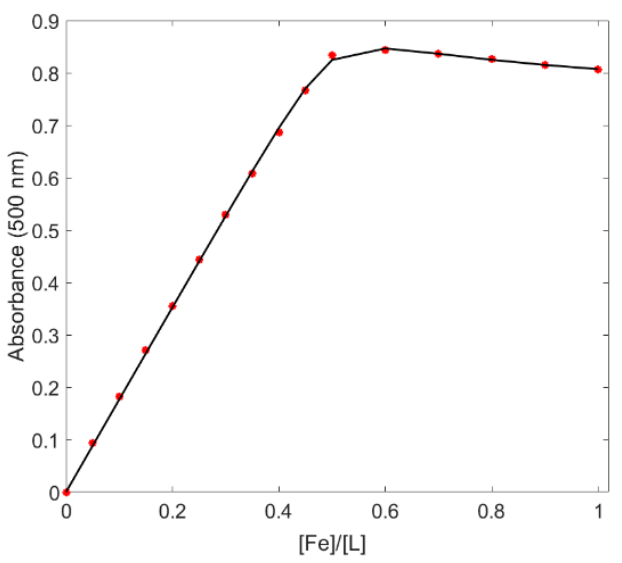

(b)

Figure 3. (a) Absorbance vs. $\left[\mathrm{Fe}^{3+}\right] /\left[\right.$ ligand] mole-ratio plot for all $\mathrm{Fe}^{3+}-\mathbf{7 d}, \mathbf{f}-\mathbf{h}, \mathbf{j}$ complexes; (b) Absorbance vs. $\left[\mathrm{Fe}^{3+}\right] /\left[\right.$ ligand] mole-ratio plot for $\mathrm{Fe}^{3+}-7 \mathrm{~d}$ complexation in aq. $\mathrm{MeOH} 85 \%$ (red asterisks-experimental data, black line-nonlinear curve-fitting according to eqn. 3 from Materials and Methods Section).

Table 1. Stoichiometry and $\mathrm{K} f$ values for $\mathrm{Fe}^{3+}-\mathbf{7 d}, \mathrm{Fe}^{3+}-\mathbf{7 f}, \mathrm{Fe}^{3+}-\mathbf{7 g}, \mathrm{Fe}^{3+}-7 \mathbf{h}$ and $\mathrm{Fe}^{3+}-7 \mathbf{j}$ complexes determined by the mole-ratio method.

\begin{tabular}{|c|c|c|c|c|}
\hline Ligand & M:L & $K_{1,2}$ & $\log K_{1,2}$ & $\lambda[\mathrm{nm}]^{\mathrm{a}}$ \\
\hline \multirow[b]{2}{*}{$7 d$} & $1: 1$ & $1.67 \times 10^{6}$ & 6.22 & \multirow[b]{2}{*}{500} \\
\hline & $1: 2$ & $2.32 \times 10^{5}$ & 5.36 & \\
\hline \multirow[b]{2}{*}{$7 f$} & $1: 1$ & $5.09 \times 10^{6}$ & 6.71 & \multirow[b]{2}{*}{500} \\
\hline & $1: 2$ & $6.04 \times 10^{5}$ & 5.78 & \\
\hline \multirow{2}{*}{$7 g$} & $1: 1$ & $2.16 \times 10^{7}$ & 7.33 & \multirow{2}{*}{500} \\
\hline & $1: 2$ & $5.38 \times 10^{4}$ & 4.73 & \\
\hline \multirow[t]{2}{*}{$7 \mathrm{~h}$} & $1: 1$ & $8.22 \times 10^{5}$ & 5.91 & \multirow[t]{2}{*}{$500,550,600$} \\
\hline & $1: 2$ & $3.39 \times 10^{5}$ & 5.53 & \\
\hline \multirow[t]{2}{*}{$7 \mathbf{j}$} & $1: 1$ & $2.31 \times 10^{5}$ & 5.36 & \multirow[t]{2}{*}{470} \\
\hline & $1: 2$ & $4.06 \times 10^{5}$ & 5.61 & \\
\hline
\end{tabular}

${ }^{\text {a }}$ Characteristic absorbance wavelength at which absorbance of the complex was measured.

\section{Materials and Methods}

\subsection{General Considerations}

All commercial reagents were used without further purification. NMR spectra were acquired using Bruker Avance III spectrometer (Billerica, MA, USA) $\left({ }^{1} \mathrm{H}: 400.13 \mathrm{MHz} ;{ }^{13} \mathrm{C}: 100.61 \mathrm{MHz}\right.$; chemical shifts are reported as parts per million $(\delta, \mathrm{ppm})$; solvents-DMSO- $d_{6}$ or $\mathrm{CDCl}_{3}$, the residual solvent peaks were used as internal standards: $2.50 \mathrm{ppm}$ for ${ }^{1} \mathrm{H}$ and $39.52 \mathrm{ppm}$ for ${ }^{13} \mathrm{C}$ (DMSO- $d_{6}$ ) or $7.26 \mathrm{ppm}$ for ${ }^{1} \mathrm{H}$ and $77.16 \mathrm{ppm}$ for ${ }^{13} \mathrm{C}\left(\mathrm{CDCl}_{3}\right)$; multiplicities are abbreviated as follows: $\mathrm{s}=$ singlet, $\mathrm{d}=$ doublet, $\mathrm{t}=$ triplet, $\mathrm{q}=$ quartet, $\mathrm{m}=$ multiplet, $\mathrm{br}=$ broad; coupling constants, $J$, are reported in Hz. Mass spectra were acquired using Bruker microTOF spectrometer (ionization by electrospray, positive ions detection; Billerica, MA, USA). Melting points were determined in open capillary tubes on Stuart SMP50 Automatic Melting Point Apparatus (Stone, UK). Homophthalic acid, hydroxylamine hydrochloride, O-benzylhydroxylamine, aldehydes, and $\mathrm{Fe}\left(\mathrm{NO}_{3}\right)_{3} \cdot 9 \mathrm{H}_{2} \mathrm{O}$ were obtained from commercial sources. All reactions were performed in air, unless otherwise noted. Analytical data obtained for compounds $7 \mathbf{a}, 7 \mathbf{b}, 7 \mathrm{k}$ and $7 \mathrm{~m}$ have been consistent with those reported in our previous work [17]. 


\subsection{Synthesis}

\subsubsection{Homophthalic Anhydride 4 (Scheme 3)}

Homophthalic acid (150 mg, $0.83 \mathrm{mmol})$ was refluxed in toluene $(15 \mathrm{~mL})$ in a flask equipped a Dean-Stark distilling trap (ISOLAB Laborgeräte $\mathrm{GmbH}$, Wertheim, Germany) for $16 \mathrm{~h}$. After cooling to room temperature, the reaction mixture was cooled to room temperature and concentrated in vacuo to provide pure title compound (135 mg, $0.83 \mathrm{mmol}$, quant. yield). ${ }^{1} \mathrm{H}-\mathrm{NMR}\left(400 \mathrm{MHz}, \mathrm{CDCl}_{3}\right) \delta 8.21$ $(\mathrm{dd}, J=7.9,1.4 \mathrm{~Hz}, 1 \mathrm{H}), 7.69(\mathrm{td}, J=7.6,1.4 \mathrm{~Hz}, 1 \mathrm{H}), 7.51(\mathrm{td}, J=7.7,1.0 \mathrm{~Hz}, 1 \mathrm{H}), 7.44-7.30(\mathrm{~m}, 1 \mathrm{H})$, $4.14(\mathrm{~s}, 2 \mathrm{H})[26]$.

\subsubsection{Hydroxylamine Acetate}

Hydroxylamine hydrochloride $(10.0 \mathrm{~g}, 144 \mathrm{mmol})$ was dissolved in deionized water $(5 \mathrm{~mL})$. The solution was added, on stirring, to a solution of sodium acetate $(11.8 \mathrm{~g}, 144 \mathrm{mmol})$ in water $(5 \mathrm{~mL})$. The resulting clear solution was concentrated to dryness. The solid residue was suspended in anhydrous methanol $(20 \mathrm{~mL})$ and filtered to remove sodium chloride. The filtrate was concentrated in vacuo to give hydroxylamine acetate $(13.0 \mathrm{~g}, 141 \mathrm{mmol}, 98 \%) .{ }^{1} \mathrm{H}-\mathrm{NMR}\left(400 \mathrm{MHz}, \mathrm{DMSO}-d_{6}\right) \delta 8.20$ (s, $\left.4 \mathrm{H}, \mathrm{NH}_{2} \mathrm{OH}, \mathrm{COOH}\right), 1.86\left(\mathrm{~s}, 3 \mathrm{H}, \mathrm{CH}_{3}\right)$ [27].

\subsubsection{General Procedure for the Preparation of N-Hydroxy THIQ Acids 7a-k}

A mixture of homophthalic acid $(180 \mathrm{mg}, 1 \mathrm{mmol})$, hydroxylamine acetate $(102 \mathrm{mg}, 1.1 \mathrm{mmol})$ and the respective aldehyde $(1.1 \mathrm{mmol})$ in toluene $(10 \mathrm{~mL})$ was heated at reflux for $24 \mathrm{~h}$ in a flask equipped a Dean-Stark distilling trap. A thick precipitate which formed on cooling the reaction mixture to $-20{ }^{\circ} \mathrm{C}$ was isolated by filtration, washed with hexane, and crystallized from aqueous methanol to provide pure title compounds.

(3R/S,4R/S)-3-(2-(Benzyloxy)phenyl)-2-hydroxy-1-oxo-1,2,3,4-tetrahydroisoquinoline-4-carboxylic acid (7c). Yield $202 \mathrm{mg}$ (52\%); white powder, mp 214.3-214.7 ${ }^{\circ} \mathrm{C} ;{ }^{1} \mathrm{H}-\mathrm{NMR}\left(400 \mathrm{MHz}, \mathrm{DMSO}-d_{6}\right) \delta 13.04$ (br.s, $1 \mathrm{H}$, $\mathrm{COOH}), 10.23$ (s, 1H, N-OH), 8.00-7.97 (m, 1H, CH(Ar)), 7.60 (d, J = 7.3 Hz, 2H, 2CH(Ar)), 7.46-7.33 $(\mathrm{m}, 5 \mathrm{H}, 5 \mathrm{CH}(\mathrm{Ar})), 7.29-7.10(\mathrm{~m}, 3 \mathrm{H}, 3 \mathrm{CH}(\mathrm{Ar})), 6.78(\mathrm{t}, J=7.5 \mathrm{~Hz}, 1 \mathrm{H}, \mathrm{CH}(\mathrm{Ar})), 6.66(\mathrm{~d}, J=7.5 \mathrm{~Hz}, 1 \mathrm{H}$, $\mathrm{CH}(\mathrm{Ar})), 5.89$ (s, 1H, 3-H), 5.34-5.15 (m, 2H, $\left.\mathrm{CH}_{2}\right), 4.19$ (s, 1H, 4-H). ${ }^{13} \mathrm{C}-\mathrm{NMR}\left(101 \mathrm{MHz}, \mathrm{DMSO}-d_{6}\right) \delta$ $172.3,161.1,155.6,137.5,133.3,132.1,130.5,129.3,128.9,128.8,128.3,128.2,127.6,127.0,126.4,126.2$, 120.7, 113.0, 69.8, 61.0, 49.9. HRMS (ESI), $m / z$ calcd for $\mathrm{C}_{23} \mathrm{H}_{20} \mathrm{NO}_{5}[\mathrm{M}+\mathrm{H}]^{+} 412.1155$, found 412.1149 .

(3R/S,4R/S)-3-(2,3-Dihydrobenzo[b][1,4]dioxin-6-yl)-2-hydroxy-1-oxo-1,2,3,4-tetrahydroisoquinoline-4carboxylic acid (7d). Yield $208 \mathrm{mg}(61 \%)$; white powder, mp 219.8-220.2 ${ }^{\circ} \mathrm{C} .{ }^{1} \mathrm{H}-\mathrm{NMR}(400 \mathrm{MHz}$, DMSO-d 6 ) $\delta 13.07$ (br.s, 1H, COOH), 10.21 (s, 1H, N-OH), 7.97 (d, J = 7.0 Hz, 1H, CH(Ar)), 7.42 (p, $J=7.4,7.0 \mathrm{~Hz}, 1 \mathrm{H}, \mathrm{CH}(\mathrm{Ar})), 7.49-7.36(\mathrm{~m}, 2 \mathrm{H}, 2 \mathrm{CH}(\mathrm{Ar})) .7 .30$ (d, J = 6.9 Hz, 1H, CH(Ar)), 6.73 (d, $J=8.1 \mathrm{~Hz}, 1 \mathrm{H}, \mathrm{CH}(\mathrm{Ar})), 6.61(\mathrm{t}, J=7.9 \mathrm{~Hz}, 1 \mathrm{H}, \mathrm{CH}(\mathrm{Ar})), 6.20(\mathrm{~d}, J=7.6 \mathrm{~Hz}, 1 \mathrm{H}, \mathrm{CH}(\mathrm{Ar})), 5.72(\mathrm{~s}, 1 \mathrm{H}$, 3-H), 4.46-4.31 (m, 2H, CH$), 4.27\left(\mathrm{t}, J=4.0 \mathrm{~Hz}, 2 \mathrm{H}, \mathrm{CH}_{2}\right), 4.16(\mathrm{~s}, 1 \mathrm{H}, \mathrm{CH}, 4-\mathrm{H}) .{ }^{13} \mathrm{C}-\mathrm{NMR}(101 \mathrm{MHz}$, DMSO- $\left.d_{6}\right) \delta 172.3,161.1,143.9,141.0,133.4,132.1,130.6,128.9,128.3,126.9,126.8,120.6,118.1,116.9$, 64.9, 64.3, 60.6, 49.8. HRMS (ESI), $m / z$ calcd for $\mathrm{C}_{18} \mathrm{H}_{16} \mathrm{NO}_{6}[\mathrm{M}+\mathrm{H}]^{+} 342.0972$, found 342.0970.

(3R/S,4R/S)-3-(2,3-Dihydrobenzo[b][1,4]dioxin-6-yl)-2-hydroxy-1-oxo-1,2,3,4-tetrahydroisoquinoline-4carboxylic acid (7d). Yield $208 \mathrm{mg}(61 \%)$; white powder, mp 219.8-220.2 ${ }^{\circ} \mathrm{C}$. ${ }^{1} \mathrm{H}-\mathrm{NMR}(400 \mathrm{MHz}$, DMSO- $d_{6}$ ) $\delta 13.07$ (br.s, 1H, COOH), 10.21 (s, 1H, N-OH), 7.97 (d, J = 7.0 Hz, 1H, CH(Ar)), 7.42 (p, $J=7.4,7.0 \mathrm{~Hz}, 1 \mathrm{H}, \mathrm{CH}(\mathrm{Ar})), 7.49-7.36(\mathrm{~m}, 2 \mathrm{H}, 2 \mathrm{CH}(\mathrm{Ar})) .7 .30$ (d, J = 6.9 Hz, 1H, CH(Ar)), 6.73 (d, $J=8.1 \mathrm{~Hz}, 1 \mathrm{H}, \mathrm{CH}(\mathrm{Ar})), 6.61(\mathrm{t}, J=7.9 \mathrm{~Hz}, 1 \mathrm{H}, \mathrm{CH}(\mathrm{Ar})), 6.20(\mathrm{~d}, J=7.6 \mathrm{~Hz}, 1 \mathrm{H}, \mathrm{CH}(\mathrm{Ar})), 5.72(\mathrm{~s}, 1 \mathrm{H}$, 3-H), 4.46-4.31 (m, 2H, CH 2$), 4.27\left(\mathrm{t}, J=4.0 \mathrm{~Hz}, 2 \mathrm{H}, \mathrm{CH}_{2}\right), 4.16(\mathrm{~s}, 1 \mathrm{H}, \mathrm{CH}, 4-\mathrm{H}) .{ }^{13} \mathrm{C}-\mathrm{NMR}(101 \mathrm{MHz}$, DMSO-d $\left.d_{6}\right) \delta 172.3,161.1,143.9,141.0,133.4,132.1,130.6,128.9,128.3,126.9,126.8,120.6,118.1,116.9$, $64.9,64.3,60.6,49.8$. HRMS (ESI), $m / z$ calcd for $\mathrm{C}_{18} \mathrm{H}_{16} \mathrm{NO}_{6}[\mathrm{M}+\mathrm{H}]^{+} 342.0972$, found 342.0970 . 
(3R/S,4R/S)-3-(4-Ethoxy-3-methoxyphenyl)-2-hydroxy-1-oxo-1,2,3,4-tetrahydroisoquinoline-4-carboxylic acid (7e). Yield $239 \mathrm{mg}(67 \%)$; white powder, mp 218.7-218.9 ${ }^{\circ} \mathrm{C} ;{ }^{1} \mathrm{H}-\mathrm{NMR}\left(400 \mathrm{MHz}, \mathrm{DMSO}-d_{6}\right) \delta 13.04(\mathrm{~s}$, 1H, COOH), 10.18 (s, 1H, N-OH), 7.94 (d, J = 7.3 Hz, 1H, CH(Ar)), 7.49-7.38 (m, 2H, 2CH(Ar)), 7.30 $(\mathrm{d}, J=7.2 \mathrm{~Hz}, 1 \mathrm{H}, \mathrm{CH}(\mathrm{Ar})), 6.80(\mathrm{~s}, 1 \mathrm{H}, \mathrm{CH}(\mathrm{Ar})), 6.78(\mathrm{~d}, J=8.4 \mathrm{~Hz}, 1 \mathrm{H}, \mathrm{CH}(\mathrm{Ar})), 6.53(\mathrm{~d}, J=8.2 \mathrm{~Hz}$, $1 \mathrm{H}, \mathrm{CH}(\mathrm{Ar})), 5.42(\mathrm{~s}, 1 \mathrm{H}, 3-\mathrm{H}), 4.28(\mathrm{~d}, J=1.8 \mathrm{~Hz}, 1 \mathrm{H}, 4-\mathrm{H}), 3.91\left(\mathrm{q}, J=6.9 \mathrm{~Hz}, 2 \mathrm{H}, \mathrm{CH}_{2}\right), 3.66(\mathrm{~s}, 3 \mathrm{H}$, $\left.\mathrm{OCH}_{3}\right), 1.26\left(\mathrm{t}, J=7.0 \mathrm{~Hz}, 3 \mathrm{H}, \mathrm{CH}_{3}\right) .{ }^{13} \mathrm{C}-\mathrm{NMR}\left(101 \mathrm{MHz}, \mathrm{DMSO}-d_{6}\right) \delta 172.3,160.7,149.2,147.8,133.6$, $132.1,131.3,130.2,129.1,128.2,126.9,118.3,113.0,110.7,65.3,64.1,55.8,51.9,15.2$. HRMS (ESI), $m / z$ calcd for $\mathrm{C}_{19} \mathrm{H}_{20} \mathrm{NO}_{6}[\mathrm{M}+\mathrm{H}]^{+} 358.1285$, found 358.1300 .

(3R/S,4R/S)-2-Hydroxy-1-oxo-3-(3,4,5-trimethoxyphenyl)-1,2,3,4-tetrahydroisoquinoline-4-carboxylic acid (7f). Yield $212 \mathrm{mg}$ (57\%); white powder, mp 220.6-220.8 ${ }^{\circ} \mathrm{C} ;{ }^{1} \mathrm{H}-\mathrm{NMR}\left(400 \mathrm{MHz}, \mathrm{DMSO}-d_{6}\right) \delta 13.09(\mathrm{~s}, 1 \mathrm{H}$, $\mathrm{COOH}), 10.24$ (s, 1H, N-OH), 7.95 (dd, J = 7.4, 1.3 Hz, 1H, CH(Ar)), 7.51-7.39 (m, 2H, 2CH(Ar)), 7.31 (d, $J=7.1 \mathrm{~Hz}, 1 \mathrm{H}, \mathrm{CH}(\mathrm{Ar})), 6.44(\mathrm{~s}, 2 \mathrm{H}, \mathrm{CH}(\mathrm{Ar})), 5.44(\mathrm{~s}, 1 \mathrm{H}, 3-\mathrm{H}), 4.33(\mathrm{~s}, 1 \mathrm{H}, 4-\mathrm{H}), 3.63\left(\mathrm{~s}, 6 \mathrm{H}, 2 \mathrm{OCH}_{3}\right)$, $3.58\left(\mathrm{~s}, 3 \mathrm{H}, \mathrm{OCH}_{3}\right) .{ }^{13} \mathrm{C}-\mathrm{NMR}\left(101 \mathrm{MHz}, \mathrm{DMSO}-d_{6}\right) \delta 172.2,160.7,153.2,137.2,134.8,133.7,132.2,130.2$, $129.0,128.3,126.8,104.1,65.5,60.3,56.2,51.8$. HRMS (ESI), $m / z$ calcd for $\mathrm{C}_{19} \mathrm{H}_{20} \mathrm{NO}_{7}[\mathrm{M}+\mathrm{H}]^{+} 374.1234$, found 374.1244

(3R/S,4R/S)-3-(2,3-Dimethoxyphenyl)-2-hydroxy-1-oxo-1,2,3,4-tetrahydroisoquinoline-4-carboxylic acid (7g). Yield $154 \mathrm{mg}$ (45\%); white powder, mp 224.3-224.6 ${ }^{\circ} \mathrm{C} ;{ }^{1} \mathrm{H}-\mathrm{NMR}\left(400 \mathrm{MHz}, \mathrm{DMSO}-d_{6}\right) \delta 13.04$ (br.s, 1H, COOH), 10.18 (s, 1H, N-OH), 7.94 (d, J = 7.1 Hz, 1H, CH(Ar)), 7.50-7.36 (m, 2H, 2CH(Ar)), 7.29 (d, $J=7.1 \mathrm{~Hz}, 1 \mathrm{H}, \mathrm{CH}(\mathrm{Ar})), 6.80(\mathrm{~s}, 1 \mathrm{H}, \mathrm{CH}(\mathrm{Ar})) 6.79(\mathrm{~d}, J=8.2 \mathrm{~Hz}, 1 \mathrm{H}, \mathrm{CH}(\mathrm{Ar})), 6.54(\mathrm{~d}, J=9.6 \mathrm{~Hz}, 1 \mathrm{H}$, $\mathrm{CH}(\mathrm{Ar})), 5.42$ (s, 1H, 4-H), $4.28(\mathrm{~s}, 1 \mathrm{H}, 3-\mathrm{H}), 3.66\left(\mathrm{~s}, 3 \mathrm{H}, \mathrm{OCH}_{3}\right), 3.65\left(\mathrm{~s}, 3 \mathrm{H}, \mathrm{OCH}_{3}\right) .{ }^{13} \mathrm{C}-\mathrm{NMR}(101 \mathrm{MHz}$, DMSO- $\left.d_{6}\right) \delta 177.1,165.5,153.9,153.4,138.4,137.0,136.1,135.0,133.8,133.0,131.66,123.1,116.7,115.4$, 70.0, 60.6, 60.6, 56.7. HRMS (ESI), $m / z$ calcd for $\mathrm{C}_{18} \mathrm{H}_{18} \mathrm{NO}_{6}[\mathrm{M}+\mathrm{H}]^{+} 344.1129$, found 344.1141.

(3R/S,4R/S)-3-(2,4-Dimethoxyphenyl)-2-hydroxy-1-oxo-1,2,3,4-tetrahydroisoquinoline-4-carboxylic acid (7h). Yield $205 \mathrm{mg}$ (60\%); white powder, mp 208.2-208.7 ${ }^{\circ} \mathrm{C} ;{ }^{1} \mathrm{H}-\mathrm{NMR}\left(400 \mathrm{MHz}, \mathrm{DMSO}-d_{6}\right) \delta 12.99$ (br.s, $1 \mathrm{H}, \mathrm{COOH}), 10.14(\mathrm{~s}, 1 \mathrm{H}, \mathrm{N}-\mathrm{OH}), 7.99-7.92(\mathrm{~m}, 1 \mathrm{H}, \mathrm{CH}(\mathrm{Ar})), 7.41$ (td, J = 7.7, 6.6, 3.9 Hz, 2H, 2CH(Ar)), $7.26(\mathrm{dd}, J=5.8,2.8 \mathrm{~Hz}, 1 \mathrm{H}, \mathrm{CH}(\mathrm{Ar})), 6.59(\mathrm{~d}, J=2.2 \mathrm{~Hz}, 1 \mathrm{H}, \mathrm{CH}(\mathrm{Ar})), 6.50$ (d, J = 8.5 Hz, 1H, CH(Ar)), $6.33(\mathrm{dd}, J=8.5,2.2 \mathrm{~Hz}, 1 \mathrm{H}, \mathrm{CH}(\mathrm{Ar})), 5.66(\mathrm{~s}, 1 \mathrm{H}, 4-\mathrm{H}), 4.05(\mathrm{~s}, 1 \mathrm{H}, 3-\mathrm{H}), 3.86\left(\mathrm{~s}, 3 \mathrm{H}, \mathrm{OCH}_{3}\right), 3.68$ (s, $\left.3 \mathrm{H}, \mathrm{OCH}_{3}\right) .{ }^{13} \mathrm{C}-\mathrm{NMR}\left(101 \mathrm{MHz}, \mathrm{DMSO}-d_{6}\right) \delta 172.5,161.1,160.6,157.6,133.5,132.0,130.4,128.9,128.2$, $126.9,126.7,118.1,104.7,99.3,60.7,56.2,55.6,50.1$. HRMS (ESI), $\mathrm{m} / \mathrm{z}$ calcd for $\mathrm{C}_{18} \mathrm{H}_{18} \mathrm{NO}_{6}[\mathrm{M}+\mathrm{H}]^{+}$ 344.1129 , found 344.1141 .

(3R/S,4R/S)-3-(2,5-Dimethoxyphenyl)-2-hydroxy-1-oxo-1,2,3,4-tetrahydroisoquinoline-4-carboxylic acid (7i). This product was isolated after washing the precipitate from r.m. with $\mathrm{Et}_{2} \mathrm{O}$ and filtration. Yield $235 \mathrm{mg}$ (54\%); white powder, mp 211.6-211.9 ${ }^{\circ} \mathrm{C} ;{ }^{1} \mathrm{H}-\mathrm{NMR}\left(400 \mathrm{MHz}, \mathrm{DMSO}-d_{6}\right) \delta 13.06$ (br.s, $1 \mathrm{H}$, $\mathrm{COOH}), 10.23$ (s, 1H, N-OH), 8.00-7.94 (m, 1H, CH(Ar)), 7.47-7.38 (m, 1H, CH(Ar)), 7.29-7.25 (m, 1H, $\mathrm{CH}(\mathrm{Ar})), 6.96$ (d, J = 8.9 Hz, 1H, CH(Ar)), 6.77 (dd, J = 8.9, 3.0 Hz, 1H, CH(Ar)), 6.19 (d, J = 2.7 Hz, 1H, CH(Ar)), 5.71 (s, 1H, 3-H), 4.08 (s, 1H, 4-H), $3.83\left(\mathrm{~s}, 3 \mathrm{H}, \mathrm{OCH}_{3}\right), 3.51\left(\mathrm{~s}, 3 \mathrm{H}, \mathrm{OCH}_{3}\right) .{ }^{13} \mathrm{C}-\mathrm{NMR}$ $\left(101 \mathrm{MHz}, \mathrm{DMSO}-d_{6}\right) \delta 172.3,161.1,153.2,150.6,133.4,132.2,130.5,128.9,128.3,127.2,126.9,113.6$, 112.5, 61.0, 56.5, 55.6, 49.8. HRMS (ESI), $m / z$ calcd for $\mathrm{C}_{18} \mathrm{H}_{18} \mathrm{NO}_{6}[\mathrm{M}+\mathrm{H}]^{+} 344.1129$, found 344.1143 .

(3R/S,4R/S)-3-(5-Chloro-2-methoxyphenyl)-2-hydroxy-1-oxo-1,2,3,4-tetrahydroisoquinoline-4-carboxylic acid (7j). Yield $249 \mathrm{mg}$ (72\%); white powder, mp 229.2-229.5 ${ }^{\circ} \mathrm{C} ;{ }^{1} \mathrm{H}-\mathrm{NMR}\left(400 \mathrm{MHz}, \mathrm{DMSO}-d_{6}\right) \delta 13.10$ (br.s, 1H, COOH), 10.35 (s, 1H, N-OH), 7.99 (d, J = 7.0 Hz, 1H, CH(Ar)), 7.48-7.40 (m, 2H, 2CH(Ar)), 7.31-7.28 (m, 1H, CH(Ar)), $7.27(\mathrm{~d}, J=2.6 \mathrm{~Hz}, 1 \mathrm{H}, \mathrm{CH}(\mathrm{Ar})), 7.08(\mathrm{~d}, J=8.8 \mathrm{~Hz}, 1 \mathrm{H}, \mathrm{CH}(\mathrm{Ar})), 6.58(\mathrm{~s}, 1 \mathrm{H}$, $\mathrm{CH}(\mathrm{Ar})), 5.72(\mathrm{~s}, 1 \mathrm{H}, 3-\mathrm{H}), 4.14(\mathrm{~s}, 1 \mathrm{H}, 4-\mathrm{H}), 3.89\left(\mathrm{~s}, 3 \mathrm{H}, \mathrm{OCH}_{3}\right) .{ }^{13} \mathrm{C}-\mathrm{NMR}\left(101 \mathrm{MHz}, \mathrm{DMSO}-d_{6}\right) \delta$ $172.15,161.05,155.59,133.20,132.35,130.56,129.08,128.58,128.50,128.11,127.02,125.92,124.34,113.67$, 60.91, 56.66, 49.35. HRMS (ESI), $m / z$ calcd for $\mathrm{C}_{17} \mathrm{H}_{15} \mathrm{ClNO}_{5}[\mathrm{M}+\mathrm{H}]^{+}$348.0633, found 348.0621. 


\subsubsection{General Procedure for the Preparation of N-Hydroxy THIQ Acids 71, $\mathbf{m}$}

A mixture of homophthalic acid $(3.6 \mathrm{~g}, 20 \mathrm{mmol})$, hydroxylamine acetate $(1.86 \mathrm{~g}, 20 \mathrm{mmol})$ and the respective aldehyde $(20 \mathrm{mmol})$ in toluene $(20 \mathrm{~mL})$ was placed in a pre-heated oil bath and refluxed for $48 \mathrm{~h}$ in a flask equipped a Dean-Stark distilling trap. A thick precipitate which formed on cooling the reaction mixture to $-20^{\circ} \mathrm{C}$ was isolated by filtration, washed with hexane, and crystallized from aqueous methanol to provide pure title compounds.

(3R/S,4R/S)3-(4-(tert-Butyl)phenyl)-2-hydroxy-1-oxo-1,2,3,4-tetrahydroisoquinoline-4-carboxylic acid (71). Yield $3.67 \mathrm{~g}$, 54\%. White solid, mp 234-236 ${ }^{\circ} \mathrm{C} ;{ }^{1} \mathrm{H}-\mathrm{NMR}\left(400 \mathrm{MHz}, \mathrm{DMSO}-d_{6}\right) \delta 13.06(\mathrm{~s}, 1 \mathrm{H})$, $10.18(\mathrm{~s}, 1 \mathrm{H}), 7.95(\mathrm{dd}, J=7.5,1.3 \mathrm{~Hz}, 1 \mathrm{H}), 7.50-7.38(\mathrm{~m}, 2 \mathrm{H}), 7.33-7.25(\mathrm{~m}, 3 \mathrm{H}), 7.05(\mathrm{~d}, J=8.3 \mathrm{~Hz}, 2 \mathrm{H})$, $5.45(\mathrm{~d}, J=1.3 \mathrm{~Hz}, 1 \mathrm{H}), 4.27(\mathrm{~d}, J=1.3 \mathrm{~Hz}, 1 \mathrm{H}), 1.21(\mathrm{~s}, 9 \mathrm{H}) .{ }^{13} \mathrm{C}-\mathrm{NMR}\left(101 \mathrm{MHz}\right.$, DMSO- $\left.d_{6}\right) \delta 172.4$, 160.7, 150.4, 136.2, 133.5, 132.2, 130.4, 129.1, 128.3, 127.0, 126.3, 125.8, 65.4, 51.8, 34.6, 31.5. HRMS (ESI), $m / z$ calcd for $\mathrm{C}_{20} \mathrm{H}_{22} \mathrm{NO}_{4}[\mathrm{M}+\mathrm{H}]^{+} 340.1543$, found 340.1553 .

\subsubsection{2-(2-Oxo-2H-chromen-3-yl)benzoic Acid 8}

Homophthalic acid (180 mg, $1 \mathrm{mmol}$ ) and 2-hydroxybenzaldehyde (122 mg, $1 \mathrm{mmol})$ were heated at reflux toluene $(15 \mathrm{~mL})$ in a flask equipped with a Dean-stark distilling trap for $24 \mathrm{~h}$. On cooling to $r$. t., the precipitate formed was separated by filtration, washed with hexane $(5 \mathrm{~mL})$, air-dried and crystallized from MeOH: $\mathrm{H}_{2} \mathrm{O}(4: 2)$ to give $194 \mathrm{mg}, 0.73 \mathrm{mmol}(73 \%)$ of the title compound as white solid: mp 259.1-259 ${ }^{\circ} \mathrm{C} ;{ }^{1} \mathrm{H}-\mathrm{NMR}\left(400 \mathrm{MHz}\right.$, DMSO- $d_{6}$ ) $\delta 12.85$ (br.s, $\left.1 \mathrm{H}, \mathrm{COOH}\right), 8.00$ (s, 1H, CH(Ar)), $7.95(\mathrm{~d}, J=7.7 \mathrm{~Hz}, 1 \mathrm{H}, \mathrm{CH}(\mathrm{Ar})), 7.79-7.73(\mathrm{~m}, 1 \mathrm{H}, \mathrm{CH}(\mathrm{Ar})), 7.69(\mathrm{td}, J=7.5,1.2 \mathrm{~Hz}, 1 \mathrm{H}, \mathrm{CH}(\mathrm{Ar}))$, 7.65-7.60 (m, 1H, CH(Ar)), $7.57(\mathrm{t}, J=7.6 \mathrm{~Hz}, 1 \mathrm{H}, \mathrm{CH}(\mathrm{Ar})), 7.50(\mathrm{~d}, J=7.5 \mathrm{~Hz}, 1 \mathrm{H}, \mathrm{CH}(\mathrm{Ar})), 7.45$ $(\mathrm{d}, J=8.2 \mathrm{~Hz}, 1 \mathrm{H}, \mathrm{CH}(\mathrm{Ar})), 7.39$ (t, $J=7.9 \mathrm{~Hz}, 1 \mathrm{H}, \mathrm{CH}(\mathrm{Ar})) .{ }^{13} \mathrm{C}-\mathrm{NMR}\left(101 \mathrm{MHz}, \mathrm{DMSO}-d_{6}\right) \delta 168.2$, 160.3, 153.4, 139.0, 136.4, 132.6, 131.9, 131.8, 131.4, 130.7, 130.2, 129.3, 128.8, 125.1, 119.9, 116.4. HRMS (ESI), $m / z$ calcd for $\mathrm{C}_{16} \mathrm{H}_{10} \mathrm{NaO}_{4}[\mathrm{M}+\mathrm{Na}]^{+}$289.0471, found 289.0461 .

\subsubsection{General Procedure for the Preparation of N-Benzyloxy THIQ Acids 9a-g}

A mixture of homophthalic acid $(3.6 \mathrm{~g}, 20 \mathrm{mmol})$, O-benzylhydroxylamine $(2.5 \mathrm{~g}, 20 \mathrm{mmol})$ and the respective aldehyde $(20 \mathrm{mmol})$ in toluene $(20 \mathrm{~mL})$ was placed in a pre-heated oil bath and refluxed for $48 \mathrm{~h}$ in a flask equipped a Dean-Stark distilling trap. A thick precipitate which formed on cooling the reaction mixture to $-20^{\circ} \mathrm{C}$ was isolated by filtration, washed with hexane, and crystallized from aqueous methanol to provide pure title compounds.

(3S/R,4S/R)-2-(Benzyloxy)-3-(4-fluorophenyl)-1-oxo-1,2,3,4-tetrahydroisoquinoline-4-carboxylic acid (9a). Yield $5.7 \mathrm{~g}, 73 \%$. White solid, mp $138-140{ }^{\circ} \mathrm{C} ;{ }^{1} \mathrm{H}-\mathrm{NMR}\left(400 \mathrm{MHz}, \mathrm{DMSO}-d_{6}\right) \delta 13.21(\mathrm{~s}, 1 \mathrm{H}), 7.98$ $(\mathrm{dd}, J=7.6,1.5 \mathrm{~Hz}, 1 \mathrm{H}), 7.51(\mathrm{td}, J=7.5,1.6 \mathrm{~Hz}, 1 \mathrm{H}), 7.45(\mathrm{ddd}, J=6.7,4.8,1.6 \mathrm{~Hz}, 3 \mathrm{H}), 7.41-7.30$ $(\mathrm{m}, 4 \mathrm{H}), 7.24(\mathrm{td}, J=8.5,6.3 \mathrm{~Hz}, 2 \mathrm{H}), 7.10(\mathrm{t}, J=8.8 \mathrm{~Hz}, 2 \mathrm{H}), 5.78(\mathrm{~d}, J=2.0 \mathrm{~Hz}, 1 \mathrm{H}), 5.07(\mathrm{~d}, J=9.9$ $\mathrm{Hz}, 1 \mathrm{H}), 5.00(\mathrm{~d}, J=10.0 \mathrm{~Hz}, 1 \mathrm{H}), 4.42(\mathrm{~d}, J=2.1 \mathrm{~Hz}, 1 \mathrm{H}) .{ }^{13} \mathrm{C}-\mathrm{NMR}\left(101 \mathrm{MHz}, \mathrm{DMSO}-d_{6}\right) \delta 171.5$, $161.5(\mathrm{~d}, J=243.9 \mathrm{~Hz}), 160.9,135.0,134.6(\mathrm{~d}, J=3.0 \mathrm{~Hz}), 133.2,132.5,129.9,129.2,128.5,128.3,128.2$ $(\mathrm{d}, J=8.2 \mathrm{~Hz}), 128.1,128.0,126.9,115.4(\mathrm{~d}, J=21.6 \mathrm{~Hz}), 75.9,62.7,51.4$. HRMS (ESI), $m / z$ calcd for $\mathrm{C}_{23} \mathrm{H}_{18} \mathrm{FNO}_{4} \mathrm{Na}[\mathrm{M}+\mathrm{Na}]^{+}$414.1112, found 414.1113.

(3S/R,4S/R)-2-(Benzyloxy)-3-(4-methoxyphenyl)-1-oxo-1,2,3,4-tetrahydroisoquinoline-4-carboxylic acid (9b). Yield $5.8 \mathrm{~g}, 54 \%$. White solid, mp $176-178{ }^{\circ} \mathrm{C} ;{ }^{1} \mathrm{H}-\mathrm{NMR}\left(400 \mathrm{MHz}\right.$, DMSO- $\left.d_{6}\right) \delta 13.15(\mathrm{~s}, 1 \mathrm{H}), 7.96(\mathrm{dd}$, $J=7.7,1.5 \mathrm{~Hz}, 1 \mathrm{H}), 7.50(\mathrm{dd}, J=7.4,1.6 \mathrm{~Hz}, 1 \mathrm{H}), 7.44(\mathrm{ddd}, J=7.1,5.2,1.7 \mathrm{~Hz}, 3 \mathrm{H}), 7.40-7.30(\mathrm{~m}, 4 \mathrm{H})$, 7.12-7.03 (m, 2H), 6.89-6.73 (m, 2H), $5.68(\mathrm{~d}, J=2.1 \mathrm{~Hz}, 1 \mathrm{H}), 5.04(\mathrm{~d}, J=9.9 \mathrm{~Hz}, 1 \mathrm{H}), 4.98(\mathrm{~d}, J=10.0 \mathrm{~Hz}$, $1 \mathrm{H}), 4.36(\mathrm{~d}, J=2.1 \mathrm{~Hz}, 1 \mathrm{H}), 3.65(\mathrm{~s}, 3 \mathrm{H}) .{ }^{13} \mathrm{C}-\mathrm{NMR}\left(101 \mathrm{MHz}, \mathrm{DMSO}-d_{6}\right) \delta 171.7,161.0,158.7,135.1$, 133.5, 132.4, 130.2, 129.9, 129.2, 128.5, 128.3, 128.2, 128.0, 127.3, 126.9, 113.9, 75.8, 63.0, 55.0, 51.6. HRMS (ESI), $m / z$ calcd for $\mathrm{C}_{24} \mathrm{H}_{21} \mathrm{NO}_{5} \mathrm{Na}[\mathrm{M}+\mathrm{Na}]^{+} 426.1312$, found 426.1318 .

(3S/R,4S/R)-2-(Benzyloxy)-3-(4-(tert-butyl)phenyl)-1-oxo-1,2,3,4-tetrahydroisoquinoline-4-carboxylic acid (9c). Yield $6.0 \mathrm{~g}, 70 \%$. White solid, mp 190-191 ${ }^{\circ} \mathrm{C} ;{ }^{1} \mathrm{H}-\mathrm{NMR}\left(400 \mathrm{MHz}\right.$, DMSO- $\left.d_{6}\right) \delta 13.19(\mathrm{~s}, 1 \mathrm{H}), 7.99$ (dd, 
$J=7.7,1.5 \mathrm{~Hz}, 1 \mathrm{H}), 7.50(\mathrm{td}, J=7.5,1.6 \mathrm{~Hz}, 1 \mathrm{H}), 7.44(\mathrm{ddd}, J=7.5,5.7,1.6 \mathrm{~Hz}, 3 \mathrm{H}), 7.39-7.31(\mathrm{~m}, 4 \mathrm{H})$, $7.28(\mathrm{~d}, J=8.5 \mathrm{~Hz}, 2 \mathrm{H}), 7.11(\mathrm{~d}, J=8.5 \mathrm{~Hz}, 2 \mathrm{H}), 5.72(\mathrm{~d}, J=2.1 \mathrm{~Hz}, 1 \mathrm{H}), 5.05(\mathrm{~d}, J=9.9 \mathrm{~Hz}, 1 \mathrm{H}), 5.01$ $(\mathrm{d}, J=9.9 \mathrm{~Hz}, 1 \mathrm{H}), 4.42(\mathrm{~d}, J=2.1 \mathrm{~Hz}, 1 \mathrm{H}), 1.18(\mathrm{~s}, 9 \mathrm{H}) .{ }^{13} \mathrm{C}-\mathrm{NMR}\left(101 \mathrm{MHz}, \mathrm{DMSO}-d_{6}\right) \delta 171.7,161.1$, 150.1, 135.4, 135.0, 133.4, 132.4, 130.0, 129.3, 128.5, 128.3, 128.2, 128.0, 126.9, 125.9, 125.4, 75.9, 63.2, 51.5, 34.1, 30.9. HRMS (ESI), $m / z$ calcd for $\mathrm{C}_{27} \mathrm{H}_{27} \mathrm{NO}_{4} \mathrm{Na}[\mathrm{M}+\mathrm{Na}]^{+} 452.1832$, found 452.1836 .

(3S/R,4S/R)-2-(Benzyloxy)-3-(4-(methoxycarbonyl)phenyl)-1-oxo-1,2,3,4-tetrahydroisoquinoline-4-carboxylic acid (9d). Yield $5.6 \mathrm{~g}, 65 \%$. White solid, mp 197-199 ${ }^{\circ} \mathrm{C} ;{ }^{1} \mathrm{H}-\mathrm{NMR}\left(400 \mathrm{MHz}, \mathrm{DMSO}-d_{6}\right) \delta 13.26(\mathrm{~s}$, $1 \mathrm{H}), 7.98(\mathrm{dd}, J=7.6,1.6 \mathrm{~Hz}, 1 \mathrm{H}), 7.92-7.77(\mathrm{~m}, 2 \mathrm{H}), 7.49(\mathrm{td}, J=7.5,1.6 \mathrm{~Hz}, 1 \mathrm{H}), 7.46-7.40(\mathrm{~m}, 3 \mathrm{H})$, $7.39-7.27(\mathrm{~m}, 6 \mathrm{H}), 5.88(\mathrm{~d}, J=1.9 \mathrm{~Hz}, 1 \mathrm{H}), 5.09(\mathrm{~d}, J=10.0 \mathrm{~Hz}, 1 \mathrm{H}), 5.03(\mathrm{~d}, J=10.0 \mathrm{~Hz}, 1 \mathrm{H}), 4.48(\mathrm{~d}$, $J=2.0 \mathrm{~Hz}, 1 \mathrm{H}), 3.78(\mathrm{~s}, 3 \mathrm{H}) .{ }^{13} \mathrm{C}-\mathrm{NMR}\left(101 \mathrm{MHz}, \mathrm{DMSO}-d_{6}\right) \delta 171.4,165.7,161.0,143.8,135.0,133.0$, 132.5, 129.9, 129.4, 129.2, 129.1, 128.5, 128.3, 128.1, 128.0, 127.0, 126.5, 76.0, 63.2, 52.1, 51.3. HRMS (ESI), $m / z$ calcd for $\mathrm{C}_{25} \mathrm{H}_{21} \mathrm{NO}_{6} \mathrm{Na}[\mathrm{M}+\mathrm{Na}]^{+} 454.1261$, found 454.1264 .

(3S/R,4S/R)-2-(Benzyloxy)-3-(4-bromophenyl)-1-oxo-1,2,3,4-tetrahydroisoquinoline-4-carboxylic acid (9e). Yield 5.1 g, 57\%. White solid, mp 187-189 ${ }^{\circ} \mathrm{C} ;{ }^{1} \mathrm{H}-\mathrm{NMR}\left(400 \mathrm{MHz}\right.$, DMSO- $\left.d_{6}\right) \delta 13.16(\mathrm{~s}, 1 \mathrm{H}), 7.97(\mathrm{dd}$, $J=7.6,1.5 \mathrm{~Hz}, 1 \mathrm{H}), 7.58-7.40(\mathrm{~m}, 6 \mathrm{H}), 7.40-7.26(\mathrm{~m}, 4 \mathrm{H}), 7.24-7.08(\mathrm{~m}, 2 \mathrm{H}), 5.76(\mathrm{~d}, J=2.0 \mathrm{~Hz}, 1 \mathrm{H}), 5.07$ $(\mathrm{d}, J=9.9 \mathrm{~Hz}, 1 \mathrm{H}), 5.00(\mathrm{~d}, J=10.0 \mathrm{~Hz}, 1 \mathrm{H}), 4.42(\mathrm{~d}, J=2.0 \mathrm{~Hz}, 1 \mathrm{H}) .{ }^{13} \mathrm{C}-\mathrm{NMR}\left(101 \mathrm{MHz}, \mathrm{DMSO}-d_{6}\right) \delta$ 171.4, 161.0, 137.9, 135.0, 133.1, 132.5, 131.5, 129.9, 129.2, 128.5, 128.4, 128.3, 128.1, 128.0, 127.0, 120.9, 75.9, 62.9, 51.3. HRMS (ESI), $m / z$ calcd for $\mathrm{C}_{23} \mathrm{H}_{18} \mathrm{BrNO}_{4} \mathrm{Na}[\mathrm{M}+\mathrm{Na}]^{+} 474.0311$, found 4754.0305.

(3S/R,4S/R)-2-(Benzyloxy)-3-(2-methylphenyl)-1-oxo-1,2,3,4-tetrahydroisoquinoline-4-carboxylic acid (9f). Yield $4.2 \mathrm{~g}, 54 \%$. White solid, mp $176-178{ }^{\circ} \mathrm{C} ;{ }^{1} \mathrm{H}-\mathrm{NMR}\left(400 \mathrm{MHz}\right.$, DMSO- $\left.d_{6}\right) \delta 13.25(\mathrm{~s}, 1 \mathrm{H}), 8.03(\mathrm{dd}$, $J=7.3,1.9 \mathrm{~Hz}, 1 \mathrm{H}), 7.54-7.43(\mathrm{~m}, 1 \mathrm{H}), 7.43-7.33(\mathrm{~m}, 5 \mathrm{H}), 7.30(\mathrm{dd}, J=6.9,1.9 \mathrm{~Hz}, 1 \mathrm{H}), 7.22(\mathrm{~d}, J=7.5$ $\mathrm{Hz}, 1 \mathrm{H}), 7.12(\mathrm{td}, J=7.4,1.3 \mathrm{~Hz}, 1 \mathrm{H}), 6.98(\mathrm{td}, J=7.6,1.4 \mathrm{~Hz}, 1 \mathrm{H}), 6.68(\mathrm{dd}, J=7.9,1.2 \mathrm{~Hz}, 1 \mathrm{H}), 5.85$ $(\mathrm{d}, J=1.8 \mathrm{~Hz}, 1 \mathrm{H}), 5.01(\mathrm{~d}, J=10.1 \mathrm{~Hz}, 1 \mathrm{H}), 4.98(\mathrm{~d}, J=10.1 \mathrm{~Hz}, 1 \mathrm{H}), 4.25(\mathrm{~d}, J=1.8 \mathrm{~Hz}, 1 \mathrm{H}), 2.44(\mathrm{~s}$, 3H). ${ }^{13}$ C-NMR (101 MHz, DMSO- $d_{6}$ ) $\delta 171.8,161.3,135.8,135.2,135.0,132.9,132.3,131.1,130.1,129.2$, $128.5,128.3,128.2,128.0,127.6,126.8,125.8,124.7,76.0,61.1,49.9,18.5$. HRMS (ESI), $m / z$ calcd for $\mathrm{C}_{24} \mathrm{H}_{21} \mathrm{NO}_{4} \mathrm{Na}[\mathrm{M}+\mathrm{Na}]^{+}$410.1363, found 410.1371.

(3S/R,4S/R)-2-(Benzyloxy)-3-(4-nitrophenyl)-1-oxo-1,2,3,4-tetrahydroisoquinoline-4-carboxylic acid (9g). Yield $3.3 \mathrm{~g}, 47 \%$. Pale yellow solid, mp 190-191 ${ }^{\circ} \mathrm{C} ;{ }^{1} \mathrm{H}-\mathrm{NMR}\left(400 \mathrm{MHz}\right.$, DMSO- $\left.d_{6}\right) \delta 13.30(\mathrm{~s}, 1 \mathrm{H}), 8.13$ $(\mathrm{d}, J=8.8 \mathrm{~Hz}, 2 \mathrm{H}), 7.99(\mathrm{dd}, J=7.6,1.6 \mathrm{~Hz}, 1 \mathrm{H}), 7.58-7.41(\mathrm{~m}, 6 \mathrm{H}), 7.40-7.30(\mathrm{~m}, 4 \mathrm{H}), 5.97(\mathrm{~d}, J=1.8$ $\mathrm{Hz}, 1 \mathrm{H}), 5.10(\mathrm{~d}, J=10.0 \mathrm{~Hz}, 1 \mathrm{H}), 5.04(\mathrm{~d}, J=10.0 \mathrm{~Hz}, 1 \mathrm{H}), 4.53(\mathrm{~d}, J=2.0 \mathrm{~Hz}, 1 \mathrm{H}) .{ }^{13} \mathrm{C}-\mathrm{NMR}(101$ MHz, DMSO- $\left.d_{6}\right) \delta 171.2,161.0,147.0,146.0,134.9,132.9,132.6,130.0,129.3,128.6,128.3,128.2,127.8$, 127.5, 127.0, 123.7, 76.0, 62.9, 51.0. HRMS (ESI), $m / z$ calcd for $\mathrm{C}_{23} \mathrm{H}_{18} \mathrm{~N}_{2} \mathrm{O}_{6} \mathrm{Na}[\mathrm{M}+\mathrm{Na}]^{+} 441.1057$, found 441.1056 .

\subsubsection{2-(Benzyloxy)-3-(4-methoxyphenyl)-3,4-dihydroisoquinolin-1(2H)-one (10)}

To a stirred solution of compound 9 a $(403 \mathrm{mg}, 1 \mathrm{mmol})$ in DMSO $(3 \mathrm{~mL})$ in a screw-cap vial $\mathrm{K}_{2} \mathrm{CO}_{3}(207 \mathrm{mg}, 1.5 \mathrm{mmol})$ was added. The mixture was heated to $110^{\circ} \mathrm{C}$ and stirred overnight. After cooling to room temperature, the reaction mixture was diluted with EtOAc $(30 \mathrm{~mL})$ and water $(15 \mathrm{~mL})$. The organic layer was then separated and washed with water $(3 \times 15 \mathrm{~mL})$, dried over $\mathrm{Na}_{2} \mathrm{SO}_{4}$, filtered, and concentrated in vacuo to give pure title compound as beige foam. Yield $275 \mathrm{mg}, 77 \% .{ }^{1} \mathrm{H}-\mathrm{NMR}$ $\left(400 \mathrm{MHz}, \mathrm{CDCl}_{3}\right) \delta 8.19(\mathrm{dd}, J=7.6,1.6 \mathrm{~Hz}, 1 \mathrm{H}), 7.44-7.35(\mathrm{~m}, 2 \mathrm{H}), 7.33(\mathrm{~s}, 5 \mathrm{H}), 7.17-7.10(\mathrm{~m}, 2 \mathrm{H})$, $7.08-7.03(\mathrm{~m}, 1 \mathrm{H}), 6.85-6.72(\mathrm{~m}, 2 \mathrm{H}), 5.15(\mathrm{~d}, J=10.3 \mathrm{~Hz}, 1 \mathrm{H}), 4.91(\mathrm{~d}, J=10.3 \mathrm{~Hz}, 1 \mathrm{H}), 4.74(\mathrm{t}, J=5.6$ $\mathrm{Hz}, 1 \mathrm{H}), 3.75(\mathrm{~s}, 3 \mathrm{H}), 3.41(\mathrm{dd}, J=16.1,6.1 \mathrm{~Hz}, 1 \mathrm{H}), 3.16(\mathrm{dd}, J=16.1,5.2 \mathrm{~Hz}, 1 \mathrm{H}) .{ }^{13} \mathrm{C}-\mathrm{NMR}(101$ $\left.\mathrm{MHz}, \mathrm{CDCl}_{3}\right) \delta 164.3,159.4,135.6(\mathrm{~d}, J=4.9 \mathrm{~Hz}), 132.6,131.1,129.8,128.8,128.8,128.5,128.2,128.1$, 127.6, 127.3, 114.1, 63.4, 55.4, 36.9. HRMS (ESI), $m / z$ calcd for $\mathrm{C}_{23} \mathrm{H}_{21} \mathrm{NO}_{3} \mathrm{Na}[\mathrm{M}+\mathrm{Na}]^{+} 382.1414$, found 382.1418 . 


\subsubsection{General Procedure for O-debenzylation}

Synthesis of Compounds 7a, 11, and $\mathbf{1 2}$

Compound $\mathbf{7 a}(100 \mathrm{mg}, 0.25 \mathrm{mmol}), \mathbf{1 0}(50 \mathrm{mg}, 0.14 \mathrm{mmol})$ or $7 \mathrm{c}(150 \mathrm{mg}, 0.38 \mathrm{mmol})$ was dissolved in $\mathrm{MeOH}(15 \mathrm{~mL}), 0.05$ molar equiv. of $10 \% \mathrm{Pd} / \mathrm{C}$ was added, and the reaction was stirred under the atmosphere of hydrogen (introduced via a balloon) for $16 \mathrm{~h}$ at room temperature. The catalyst was removed by filtration of the reaction mixture through a pad of Celite and the filtrate was concentrated in vacuo to give pure title compounds.

2-Hydroxy-3-(4-methoxyphenyl)-3,4-dihydroisoquinolin-1(2H)-one (11). Yield $35 \mathrm{mg}$, 94\%. White solid, mp 95-96 ${ }^{\circ} \mathrm{C}(\mathrm{MeOH}) ;{ }^{1} \mathrm{H}-\mathrm{NMR}\left(400 \mathrm{MHz}, \mathrm{DMSO}-d_{6}\right) \delta 9.92(\mathrm{~s}, 1 \mathrm{H}), 7.93(\mathrm{dd}, J=7.6,1.5 \mathrm{~Hz}, 1 \mathrm{H})$, $7.42(\mathrm{td}, J=7.4,1.5 \mathrm{~Hz}, 1 \mathrm{H}), 7.35(\mathrm{t}, J=7.4 \mathrm{~Hz}, 1 \mathrm{H}), 7.20(\mathrm{~d}, J=7.4 \mathrm{~Hz}, 1 \mathrm{H}), 7.09(\mathrm{~d}, J=8.7 \mathrm{~Hz}, 2 \mathrm{H})$, $6.82(\mathrm{~d}, J=8.7 \mathrm{~Hz}, 2 \mathrm{H}), 5.07(\mathrm{dd}, J=6.4,3.8 \mathrm{~Hz}, 1 \mathrm{H}), 3.77-3.48(\mathrm{~m}, 4 \mathrm{H}), 3.21-3.09(\mathrm{~m}, 1 \mathrm{H}) .{ }^{13} \mathrm{C}-\mathrm{NMR}$ $\left(101 \mathrm{MHz}\right.$, DMSO- $\left.d_{6}\right) \delta 161.4,158.5,135.4,131.9,131.8,128.6,127.8,127.4,126.8,126.7,113.7,62.5,55.0$, 36.0. HRMS (ESI), $m / z$ calcd for $\mathrm{C}_{32} \mathrm{H}_{30} \mathrm{~N}_{2} \mathrm{O}_{6} \mathrm{Na}[2 \mathrm{M}+\mathrm{Na}]^{+} 561.1996$, found 561.1994.

(3R/S,4R/S)3-(2-Hydroxyphenyl)-1-oxo-1,2,3,4-tetrahydroisoquinoline-4-carboxylic acid (12). Yield $100 \mathrm{mg}$, 93\%. White solid, mp 202.3-202. ${ }^{\circ} \mathrm{C} ;{ }^{1} \mathrm{H}-\mathrm{NMR}\left(400 \mathrm{MHz}, \mathrm{DMSO}-d_{6}\right) \delta 12.79(\mathrm{~s}, 1 \mathrm{H}, \mathrm{COOH}), 9.85$ (s, $1 \mathrm{H}, \mathrm{OH}), 8.24(\mathrm{~d}, J=4.9 \mathrm{~Hz}, 1 \mathrm{H}, \mathrm{NH}), 7.91(\mathrm{dd}, J=7.2,1.9 \mathrm{~Hz}, 1 \mathrm{H}, \mathrm{CH}(\mathrm{Ar})), 7.39(\mathrm{td}, J=7.1,1.7 \mathrm{~Hz}, 2 \mathrm{H}$, 2CH(Ar)), $7.22(\mathrm{~d}, J=7.0,1 \mathrm{H}, \mathrm{CH}(\mathrm{Ar})), 7.00(\mathrm{t}, J=8.4 \mathrm{~Hz}, 1 \mathrm{H}, \mathrm{CH}(\mathrm{Ar})) .6 .81(\mathrm{~d}, J=8.1 \mathrm{~Hz}, 1 \mathrm{H}, \mathrm{CH}(\mathrm{Ar}))$, $6.71(\mathrm{~d}, J=7.5 \mathrm{~Hz}, 1 \mathrm{H}, \mathrm{CH}(\mathrm{Ar})), 6.59(\mathrm{t}, J=7.5 \mathrm{~Hz}, 1 \mathrm{H}, \mathrm{CH}(\mathrm{Ar})), 5.39(\mathrm{dd}, J=5.0,1.7 \mathrm{~Hz}, 1 \mathrm{H}, 3-\mathrm{H}), 4.07$ (s, 1H, 4-H). ${ }^{13} \mathrm{C}-\mathrm{NMR}\left(101 \mathrm{MHz}\right.$, DMSO- $\left.d_{6}\right) \delta 173.0,164.8,154.4,135.4,132.2,130.1,129.4,128.5,128.0$, $127.7,126.9,126.9,119.0,115.6,50.8,48.6$. HRMS (ESI), $m / z$ calcd for $\mathrm{C}_{16} \mathrm{H}_{14} \mathrm{NO} 4[\mathrm{M}+\mathrm{H}]^{+}$284.0917, found 284.0924 .

\subsection{Spectrophotometric Investigation of Compounds $7 \mathrm{~d}, 7 \mathrm{f}, 7 \mathrm{~g}, 7 \mathrm{~h}, 7 \mathrm{j}$, and Their Complexation with Iron(III) Nitrate}

Spectrophotometric measurements were performed on a UV-1800 Shimadzu double beam spectrophotometer (Kyoto, Japan) using $10.00 \mathrm{~mm}$ quartz cells. Methanol or methanol—water (85:15) mixture were used as solvent. All measurements were performed at room temperature $\left(26^{\circ} \mathrm{C}\right)$ in stoppered cells. For experiments with $\mathrm{Fe}^{3+}$ complexation, $\mathrm{Fe}\left(\mathrm{NO}_{3}\right)_{3} \cdot 9 \mathrm{H}_{2} \mathrm{O}$ was used as iron source.

UV-Vis spectra of compounds $\mathbf{7 d}, \mathbf{7 f}, \mathbf{7 g}, \mathbf{7 h}, \mathbf{7 j}$ (Figure 2a and ESI, Figures S1-S3) were recorded for $\mathrm{MeOH}$ solutions $\left(\mathrm{C}=5 \times 10^{-5} \mathrm{M}\right)$ prior to titration experiments. Sample preparation for UV-Vis titrations (Figure 3a and ESI, Figures S4-S8): a $1500 \mu \mathrm{L}$ aliquote of $0.001 \mathrm{M}$ stock solution of compound $\mathbf{7 d}, \mathbf{7 f}, \mathbf{7 g}, \mathbf{7 h}, \mathbf{7 j}\left(1.5 \times 10^{-6} \mathrm{~mol}\right)$ in $\mathrm{MeOH}$ was placed in a $10.00 \mathrm{~mm}$ quartz cuvette equipped with magnetic stir bar and diluted to $3 \mathrm{~mL}$ with $1050 \mu \mathrm{L}$ of $\mathrm{MeOH}$ and $450 \mu \mathrm{L}$ of water to obtain solution in $85 \%$ aq. $\mathrm{MeOH}\left(\mathrm{C}_{\mathrm{L}}=5 \times 10^{-4} \mathrm{M}\right)$. Aliquotes, 5 or $10 \mu \mathrm{L}$ of $0.015 \mathrm{M}$ aqueous $\mathrm{Fe}\left(\mathrm{NO}_{3}\right)_{3}$ solution, were added to the cell with calibrated micropipette in a stepwise manner $\left(C_{M}=0.25-5 \times 10^{-4} \mathrm{M}\right)$. The solution was vigorously stirred after addition of each aliquote followed by registration of absorbance spectrum over the wavelength range of $300-700 \mathrm{~nm}$ vs. $85 \%$ aq. $\mathrm{MeOH}$ (all measurements were performed in stoppered cuvettes). Color of the solution was changed from colorless to purple, which corresponds to appearance of a new absorption band with maximum at $500 \mathrm{~nm}$. The absorbance at selected wavelength was plotted as a function of $\left[\mathrm{Fe}^{3+}\right] /[$ ligand] ratio to give binding isotherms presented on Figure $3 \mathrm{~b}$ and ESI, Figures S4-S8. The maxima of these curves correspond to the maximum formation of complexes and indicate to the stoichiometry of the complexes. The average stoichiometry of complex is estimated from the point where this curve changes its slope (this point is the intersect point of bilinear fitting of experimental curve). 1:2 metal-to-ligand ratio was observed in all cases. Formation constants (Table 1) were calculated from curves on Figure 3a using following equations:

$$
K=\frac{\left[M L_{x}\right]}{[M][L]^{x}}
$$




$$
\begin{gathered}
K_{1} K_{2}[L]^{3}+K_{1}\left(1+K_{2}\left(2 C_{M}-C_{L}\right)\right)[L]^{2}+\left(1+K_{1}\left(C_{M}-C_{L}\right)\right)[L]=0 \\
\Delta A_{o b s}=\frac{\varepsilon_{\Delta M L}\left(C_{M}\right) K_{1}[L]+2 \varepsilon_{\Delta M L 2}\left(C_{M}\right) K_{1} K_{2}[L]^{2}}{1+K_{1}[L]+K_{1} K_{2}[L]^{2}}
\end{gathered}
$$

Experimental curves were fitted to equation 3 corresponding to 1:2 metal-to-ligand complex formation using nonlinear curve-fitting performed in ThordarsonFittingProgram [25]. The program is based on the iterative adjustment of calculated values of absorbance (A) to observed values using Equation (3) previously derived [25] from Equations (1) and (2), where $K_{1}$ and $K_{2}$ are stepwise formation constants; $\varepsilon_{\Delta \mathrm{ML}}=\varepsilon_{\mathrm{ML}}-\varepsilon_{\mathrm{L}}$ and $\varepsilon_{\Delta \mathrm{ML} 2}=\varepsilon_{\mathrm{ML} 2}-\varepsilon_{\mathrm{ML}}$, where $\varepsilon_{i}$ are molar absorptivities of corresponding species; $\mathrm{C}_{\mathrm{L}}$ and $\mathrm{C}_{\mathrm{M}}$ are analytical concentrations of ligand and $\mathrm{Fe}^{3+}$ respectively and $\mathrm{L}$ is free ligand concentration.

\section{Conclusions}

In summary, we have developed a practically convenient, three-component approach to $\mathrm{N}$-hydroxytetrahydroisoquinoline (THIQ) acids via a variant of the Castagnoli-Cushman reaction involving in situ cyclodehydration of homophthalic acid with concomitant formation of an oxime in refluxing toluene. Using hydroxylamine acetate or O-benzylhydroxylamine in lieu of the hydroxylamine hydrochloride typically employed to prepare oximes was key to the success of the reaction. For prepared $N$-bezyloxy THIQ acids decarboxylation and debenzylation reactions were additionally investigated. Five selected cyclic hydroxamic acid compounds produced in the course of this study have been profiled and confirmed as ligands for $\mathrm{Fe}^{3+}$. Thus, a new, practically simple and flexible approach to potential iron overload disease treatments or analogs of bacterial siderophores for antibiotic delivery has been developed.

Supplementary Materials: The following are available online at http:/ /www.mdpi.com/1420-3049/24/5/864/s1,

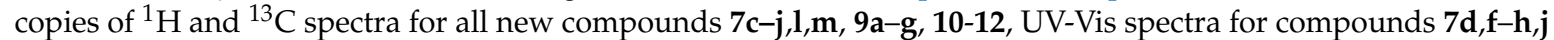
(Figures S1-S3), results of UV-Vis titration of compounds $\mathbf{7 d}, \mathbf{f}-\mathbf{h}, \mathbf{j}$ with $\mathrm{FeCl}_{3}$ (Figure S4-S8).

Author Contributions: Organic synthesis and compound characterization, E.C. and O.B.; UV-Vis spectroscopy, O.B.; conceptualization and project administration, M.K. and D.D.; methodology and validation, D.D., E.C. and O.B.; Writing-Original Draft preparation, M.K. and O.B.; Writing-Review and Editing, D.D.; funding acquisition, O.B.

Funding: This research was funded by Russian Foundation for Basic Research (RFBR), grant number 18-33-00016.

Acknowledgments: We thank the Educational Resource Center of Chemistry, Research Center for Magnetic Resonance and the Center for Chemical Analysis and Materials Research of Saint Petersburg State University Research Park for obtaining the analytical data.

Conflicts of Interest: The authors declare no conflict of interest. The funders had no role in the design of the study; in the collection, analyses, or interpretation of data; in the writing of the manuscript, or in the decision to publish the results.

\section{References}

1. Farkas, E.; Enyedy, É.A.; Micera, G.; Garribba, E. Coordination modes of hydroxamic acids in copper(II), nickel(II) and zinc(II) mixed-ligand complexes in aqueous solution. Polyhedron 2000, 19, 1727-1736. [CrossRef]

2. Schalk, I.J.; Hannauer, M.; Braud, A. New roles for bacterial siderophores in metal transport and tolerance. Environ. Microbiol. 2011, 13, 2844-2854. [CrossRef] [PubMed]

3. Ferreira, K.; Hu, H.-Y.; Fetz, V.; Prochnow, H.; Rais, B.; Müller, P.P.; Brönstrup, M. Multivalent Siderophore-DOTAM Conjugates as Theranostics for Imaging and Treatment of Bacterial Infections. Angew. Chem. Int. Ed. 2017, 56, 8272-8276. [CrossRef] [PubMed]

4. Italia, K.; Colah, R.; Ghosh, K. Experimental animal model to study iron overload and iron chelation and review of other such models. Blood Cells Mol. Dis. 2015, 55, 194-199. [CrossRef] [PubMed]

5. Liu, Y.; Jacobs, H.K.; Gopalan, A.S. A new approach to cyclic hydroxamic acids: Intramolecular cyclization of N-benzyloxy carbamates with carbon nucleophiles. Tetrahedron 2011, 67, 2206-2214. [CrossRef] [PubMed] 
6. Banerjee, R.; King, S.B. Synthesis of Cyclic Hydroxamic Acids through-NOH Insertion of Ketones. Organic Lett. 2009, 11, 4580-4583. [CrossRef] [PubMed]

7. Jewula, P.; Berthet, J.-C.; Chambron, J.-C.; Rousselin, Y.; Thuéry, P.; Meyer, M. Synthesis and Structural Study of Tetravalent $\left(\mathrm{Zr}^{4+}, \mathrm{Hf}^{4+}, \mathrm{Ce}^{4+}, \mathrm{Th}^{4+}, \mathrm{U}^{4+}\right)$ Metal Complexes with Cyclic Hydroxamic Acids. European J. Inorganic Chem. 2015, 2015, 1529-1541. [CrossRef]

8. González-López, M.; Shaw, J.T. Cyclic Anhydrides in Formal Cycloadditions and Multicomponent Reactions. Chem. Rev. 2009, 109, 164-189. [CrossRef] [PubMed]

9. Dar'in, D.; Bakulina, O.; Chizhova, M.; Krasavin, M. New Heterocyclic Product Space for the Castagnoli-Cushman Three-Component Reaction. Organic Lett. 2015, 17, 3930-3933. [CrossRef] [PubMed]

10. Castagnoli, N. Condensation of succinic anhydride with $N$-benzylidene- $N$-methylamine. Stereoselective synthesis of trans- and cis-1-methyl-4-carboxy-5-phenyl-2-pyrrolidinone. J. Organic Chem. 1969, 34, 3187-3189. [CrossRef]

11. Cushman, M.; Castagnoli, N. Novel approach to the synthesis of nitrogen analogs of the tetrahydrocannabinols. J. Organic Chem. 1973, 38, 440-448. [CrossRef]

12. Mohammadi, M.H.; Mohammadi, A.A. One-Pot, Three-Component Synthesis of Cis-Isoquinolonic Acids Using $\mathrm{ZnCl}_{2}, \mathrm{AlCl}_{3}-\mathrm{SiO}_{2}$ as Catalyst. Synth. Commun. 2011, 41, 523-527. [CrossRef]

13. Wang, L.; Liu, J.; Tian, H.; Qian, C.; Sun, J. One-Pot Synthesis of cis-Isoquinolonic Acid Derivatives via Three-Component Reaction of Homophthalic Anhydride with Aldehydes and Amines using Ytterbium(III) Triflate as Catalyst. Adv. Synth. Cataly. 2005, 347, 689-694. [CrossRef]

14. Pommier, Y. DNA Topoisomerase I Inhibitors: Chemistry, Biology and Interfacial Inhibition. Chem. Rev. 2009, 109, 2894-2902. [CrossRef] [PubMed]

15. Floyd, D.M.; Stein, P.; Wang, Z.; Liu, J.; Castro, S.; Clark, J.A.; Connelly, M.; Zhu, F.; Holbrook, G.; Matheny, A.; et al. Hit-to-Lead Studies for the Antimalarial Tetrahydroisoquinolone Carboxanilides. J. Med. Chem. 2016, 59, 7950-7962. [CrossRef] [PubMed]

16. Humphries, P.S.; Benbow, J.W.; Bonin, P.D.; Boyer, D.; Doran, S.D.; Frisbie, R.K.; Piotrowski, D.W.; Balan, G.; Bechle, B.M.; Conn, E.L.; et al. Synthesis and SAR of 1,2,[3,4-tetrahydroisoquinolin-1-ones as novel G-protein-coupled receptor 40 (GPR40) antagonists. Bioorg. Med. Chem. Lett. 2009, 19, 2400-2403. [CrossRef] [PubMed]

17. Bakulina, O.; Bannykh, A.; Dar'in, D.; Krasavin, M. Cyclic Hydroxamic Acid Analogues of Bacterial Siderophores as Iron-Complexing Agents prepared through the Castagnoli-Cushman Reaction of Unprotected Oximes. Chem. A Eur. J. 2017, 23, 17667-17673. [CrossRef] [PubMed]

18. Chupakhin, E.; Dar'in, D.; Krasavin, M. The Castagnoli-Cushman reaction in a three-component format. Tetrahedron Lett. 2018, 59, 2595-2599. [CrossRef]

19. Bogdanov, M.G.; Mitrev, Y.; Tiritiris, I. New Highly Diastereoselective Perkin/Michael Addition Domino Reaction between Homophthalic Anhydride and Aromatic Aldehydes: A Facile Approach to Blue-Fluorescent Dibenzo[c,h]chromenones. Eur. J. Organic Chem. 2010, 2011, 377-384. [CrossRef]

20. Senadi, G.C.; Mutra, M.R.; Lu, T.-Y.; Wang, J.-J. Oximes as reusable templates for the synthesis of ureas and carbamates by an in situ generation of carbamoyl oximes. Green Chem. 2017, 19, 4272-4277. [CrossRef]

21. Svinyarov, I.; Bogdanov, M.G. One-pot synthesis and radical scavenging activity of novel polyhydroxylated 3-arylcoumarins. European J. Med. Chem. 2014, 78, 198-206. [CrossRef] [PubMed]

22. Tutov, A.; Bakulina, O.; Dar'in, D.; Krasavin, M. Concise synthesis of 2-N-hydroxy-3,4-dihydroisoquinol2-one: A bacterial siderophore and human 5-lipooxygenase inhibitor. Tetrahedron Lett. 2018, 59, 1511-1512. [CrossRef]

23. Gräfe, U.; Ritzau, M.; Ihn, W.; Möllmann, U.; Fleck, W.F.; Groth, J.; Reissbrodt, R. A new microbial isoquinoline iron chelator from Streptomyces spec. 2002-104. J. Basic Microbiol. 1994, 34, 351-355. [CrossRef]

24. Schalk, I.J. A Trojan-Horse Strategy Including a Bacterial Suicide Action for the Efficient Use of a Specific Gram-Positive Antibiotic on Gram-Negative Bacteria. J. Med. Chem. 2018, 61, 3842-3844. [CrossRef] [PubMed]

25. Thordarson, P. Determining association constants from titration experiments in supramolecular chemistry. Chem. Soc. Rev. 2011, 40, 1305-1323. [CrossRef] [PubMed] 
26. Özcan, S.; Balci, M. The chemistry of homophthalic acid: A new synthetic strategy for construction of substituted isocoumarin and indole skeletons. Tetrahedron 2008, 64, 5531-5540. [CrossRef]

27. Shamshina, J.L.; Barber, P.S.; Gurau, G.; Griggs, C.S.; Rogers, R.D. Pulping of Crustacean Waste Using Ionic Liquids: To Extract or Not To Extract. ACS Sustainable Chem. Eng. 2016, 4, 6072-6081. [CrossRef]

Sample Availability: Samples of the compounds $7 \mathbf{a}-\mathbf{m}, \mathbf{9 a}-\mathbf{g}$ are available from the authors.

(C) 2019 by the authors. Licensee MDPI, Basel, Switzerland. This article is an open access article distributed under the terms and conditions of the Creative Commons Attribution (CC BY) license (http:/ / creativecommons.org/licenses/by/4.0/). 